\title{
Spectral Deep Learning for Prediction and Prospective Validation of Functional Groups
}

\author{
Jonathan Fine, Anand Rasjashekar, Krupal P. Jethava, Gaurav Chopra
}

Submitted date: $24 / 01 / 2020$ - Posted date: 24/01/2020

Licence: CC BY 4.0

Citation information: Fine, Jonathan; Rasjashekar, Anand; Jethava, Krupal P.; Chopra, Gaurav (2019): Spectral Deep Learning for Prediction and Prospective Validation of Functional Groups. ChemRxiv. Preprint. https://doi.org/10.26434/chemrxiv.8081924.v2

State-of-the-art identification of the functional groups present in an unknown chemical entity requires expertise of a skilled spectroscopist to analyse and interpret Fourier Transform Infra-Red (FTIR), Mass Spectroscopy (MS) and/or Nuclear Magnetic Resonance (NMR) data. This process can be time-consuming and error-prone, especially for complex chemical entities that poorly characterized in the literature, or inefficient to use with synthetic robots producing molecules at an accelerated rate. Herein, we introduce a fast, multi-label deep neural network for accurately identifying all the functional groups of unknown compounds using a combination of FTIR and MS spectra. We do not use any database, pre-established rules, procedures, or peak-matching methods. Our trained neural network reveals patterns typically used by human chemists to identify standard groups. Finally, we experimentally validated our neural network, trained on single compounds, to predict functional groups in compound mixtures. Our methodology showcases practical utility for future use in autonomous analytical detection.

File list (3)

Supporting - Spectra Deep Learning.pdf (1.01 MiB) view on ChemRxiv - download file

Manuscript - Spectra Deep Learning.pdf (1.36 MiB) view on ChemRxiv • download file ListingS1.xlsx (5.45 MiB) view on ChemRxiv - download file 


\section{Supporting Information}

Spectral deep learning for prediction and prospective validation of functional groups

Jonathan A. Fine ${ }^{1,{ }^{\ddagger}}$, Anand A. Rajasekar ${ }^{2,}{ }^{\ddagger}$, Krupal P. Jethava ${ }^{1}$, Gaurav Chopra ${ }^{1,3,4,5,6,7 *}$

${ }^{1}$ Department of Chemistry, Purdue University, 720 Clinic Drive, West Lafayette, IN 47906

${ }^{2}$ Indian Institute of Technology Madras

${ }^{3}$ Purdue Institute for Drug Discovery

${ }^{4}$ Purdue Center for Cancer Research

${ }^{5}$ Purdue Institute for Inflammation, Immunology and Infectious Disease

${ }^{6}$ Purdue Institute for Integrative Neuroscience

${ }^{7}$ Integrative Data Science Initiative

${ }^{\ddagger}$ These authors share an equal contribution to this work.

*Corresponding Author

E-mail: gchopra@purdue.edu 


\section{Additional Experimental Details}

\section{Standardization of IR spectra}

All IR spectra obtained from NIST was truncated so that only peaks occurring from $400 \mathrm{~cm}^{-1}$ to $4000 \mathrm{~cm}^{-1}$ remain. The IR spectra available in the NIST webbook has varying degrees of resolution, which is problematic for a multilayer perceptron (MLP) network as this architecture requires a discrete and consistent number of input dimensions. To address this, we standardized all IR spectra so that each spectrum would have the same number of peaks by defining an IR dimension as being the percent transmittance in a wavenumber bin. For example, if a compound has a transmittance of $30 \%$ between $400 \mathrm{~cm}^{-1}$ and $401 \mathrm{~cm}^{-1}$, then this dimension has a value of 0.30 . Since the most common resolution present in the NIST data is approximately $3.25 \mathrm{~cm}^{-1}$, we decided to use this resolution to standardize all IR spectra in our dataset. However, other ranges may be better suited for the problem at hand, and the selection of an optimized resolution remains as to be done as future work. To standardize all the spectra, we performed linear interpolation on each IR spectra and evaluated the fitted function at the same set of discrete points throughout each interpolated IR spectra. This process yielded uniform IR spectra consisting of 1108 points, regardless of the resolution of the original IR data.

\section{Standardization of MS spectra}

Given the discrete nature of mass spectra, the standardization process for this type of spectra is straightforward. The bin size for these spectra was chosen to be $1 \mathrm{~m} / \mathrm{z}$ unit, and the counts present in each bin were averaged together for spectra with a resolution less than $1 \mathrm{~m} / \mathrm{z}$. All the bin counts in each spectrum were divided by the largest count in the same spectrum to yield the relative abundance for all the $\mathrm{m} / \mathrm{z}$ peaks present in the dataset.

\section{Training and testing of neural networks}

All Neural Networks reported in this work were created using the Keras Python Package ${ }^{[7]}$. All hidden layers were normalized using batch normalization and activated using rectified linear units, and a sigmoidal function is used to activate the final output layer. During the training procedure, we applied a dropout procedure which randomly selects neurons given a per layer probability and sets their value to zero. We used binary cross entropy was used as the loss function for training the neural network as this loss function is standard for multi-label classification problems. For each epoch of training, Keras calculated the loss of the training and validation sets and compared this loss to the loss of the previous epoch. Early stopping with a patience of 5 epochs was used to prevent the model from overtraining. All models were validated using 5-fold cross-validation, and sequential hyperparameter searching was used to optimize the final IR and MS model. The hyperparameters of the optimized model are given in the supporting information under Details of neural networks.

The overall mathematical representation of this model can be represented with Formula 1 given below.

$\vec{y}=f\left(\overrightarrow{a^{0}}, \boldsymbol{W}, \overrightarrow{\boldsymbol{b}}\right)$

Here,,$\vec{y}$ is the predicted functional groups from the model with a length equal to the number of functional groups defined in the previous section. Each component of this vector represents the probability that the 
corresponding functional group is present in the molecule. The vector $\overrightarrow{a^{0}}$ is the input spectra and has a length equal to the number of components in the spectra. Matrix $\boldsymbol{W}$ is a weighting matrix and $\overrightarrow{\boldsymbol{b}}$ is bias a vector. All terms are applied in the following manner:

$f\left(\overrightarrow{a^{0}}, \boldsymbol{W}, \overrightarrow{\boldsymbol{b}}\right)=\sigma\left(\boldsymbol{W} \overrightarrow{a^{0}}+\overrightarrow{\boldsymbol{b}}\right)$

This function can be applied. multiple times with matrices of varying length, producing hidden 'layers.' The optimal number of layers, as well as their respective lengths, are 'hyperparameters.' For each neuron $\mathrm{k}$ in a layer I:

$a_{j}^{l}=\sigma\left(\sum_{k} \boldsymbol{w}_{j k}^{l} a_{k}^{l-1}+b_{j}^{l}\right)=\sigma\left(z_{j}^{l}\right)$

Here, $\sigma$ is an activation function. For hidden layers:

$\sigma=\operatorname{ReLU}(x)=\max (0, x)$

For the final layer:

$\sigma=\operatorname{sigmoid}(x)$

The cost function, or error in the model, is defined as follows using the binary cross entropy function:

$C(\vec{y}, \tilde{y})=\tilde{y} \log \vec{y}+(1-\tilde{y}) \tilde{y} \log (1-\vec{y})$

Where $\vec{y}$ is the predicted functional groups and $\vec{y}$ are the true functional groups. The goal of back propagation is to minimize the cost function, thereby making the model increasingly accurate.

We define the error of any neuron to be

$\delta_{j}^{l}=\frac{\delta C}{\delta z_{j}^{l}}$

For the final layer of the model, we can compute this value via the chain rule:

$\delta_{j}^{L}=\frac{\delta C}{\delta a_{j}^{L}} \sigma^{\prime}\left(z_{j}^{L}\right)$

This expression can be rewritten as the following for a matrix operation:

$\delta^{L}=\nabla_{a} C \odot \sigma^{\prime}\left(z^{L}\right)$

Once the derivative of the final layer is obtained, the derivative of the penultimate layer can be calculated as follows:

$\delta^{l}=\left(\left(\boldsymbol{W}^{l+1}\right)^{T} \delta^{l+1}\right) \odot \sigma^{\prime}\left(z^{l}\right)$

One can continue to 'backpropagate' this derivative until the derivative of the first layer is calculated. Now that $\delta_{j}^{l}$ can be calculated for all layers, the derivate of the total cost function with respect to a bias term can be written as

$\frac{\delta C}{\delta b_{j}^{l}}=\delta_{j}^{l}$ 
Additionally, the following for the weighting terms:

$\frac{\delta C}{\delta \boldsymbol{W}_{j k}^{l}}=a_{k}^{l-1} \delta_{j}^{l}$

Now that all backpropagation terms can be calculated for all layers, we can define a modified version of this procedure referred to as guided backpropagation. This procedure begins with an already-trained network consisting of $\boldsymbol{W}$ and $\overrightarrow{\boldsymbol{b}}$. The network is predicted in the forward direction to obtain $\vec{y}$. Then equations (9) and (10) are used to calculate the weights of the input vector $\overrightarrow{a^{0}}$. Note that all negative gradients are set to 0 during the application of (10). Details for the application of guided backpropagation in this work are given in the following section.

\section{Use of backpropagation to identify patterns in the model}

One of the most challenging problems of developing deep learning models is interpreting them in a chemical context ${ }^{[8]}$, which results in many researchers treating these models as black-box representations, thereby neglecting to understand how features are used to predict results. Solving this problem to understand how various input features result in a given result is a challenging problem. Many new methods were proposed to address this problem which can be broadly classified into perturbation-based methods and backpropagation-based methods. Back propagation-based methods compute the gradient of the activations concerning the feature space and identify the section of the image which maximally activates that neuron. One such backpropagation based method is 'guided backpropagation ${ }^{[9]}$.' Guided backpropagation is similar to the 'probability' approach ${ }^{[10]}$, where the difference between these two methods arises from differences in the handling of backpropagation. The deconvnet approach computes the gradient-based on top gradient signal setting negative values to zero while backpropagation sets the gradient of negative activations in the forward pass to zero; avoiding the 'flow' of negative gradients during backpropagation. We use this technique to identify the top 5 bins of an input IR spectra that was responsible for predicting a particular functional group of a molecule and present these results in Figure 4.

\section{General Information:}

IR spectra were recorded by ATR-IR method using Thermo Nicolet FTIR instrument and processed using OMNIC software. The compound mixtures were prepared by mixing two solid compounds (crushed with spatula to make fine powder, if necessary) and used directly for ATR-IR. MS spectra were recorded by Electron Spray Ionization method (ESI) using Agilent 6470 triple quadruple LC/MS. Solvents and commercially available compounds were used as it is without any further purification.

\section{Synthetic Models}

We developed a control, which we have termed as 'synthetic models', for this work which are created using a predefined accuracy to assign functional groups. To generate a synthetic model, one takes the original functional group matrix (where columns are functional groups and rows are molecules) and predicts each functional group for every molecule individually based on random numbers. The accuracy of each synthetic model is fixed, and the predictions are randomly assigned as correct or incorrect to obtain the specified accuracy. Unlike a truly random model, the synthetic model has access to the original functional group assignment matrix and the predictions of the matrix are not randomly assigned but are instead 'purposefully' correct or incorrect based on a uniform random distribution. For example, consider 
a synthetic model that has an accuracy of $50 \%$ and is being generated for 4 functional groups. It is given a molecule where only the first 2 functional groups are present $([1,1,0,0])$. Four random numbers are generated using a uniform distribution, e.g.: $0.25,0.75,0.85$, and 0.10 . Since the second and third random numbers are greater than the assigned accuracy (0.50), they are deemed incorrect and the model will predict $([1,0,1,0])$. This example has a molecular recall of 0.5 , a molecular precision of 0.5 and molecular perfection of 0 .

Synthetic models with accuracies of $99 \%, 95 \%$, and $90 \%$ are given below showing decrease in MPR with increase in number of functional group predictions.
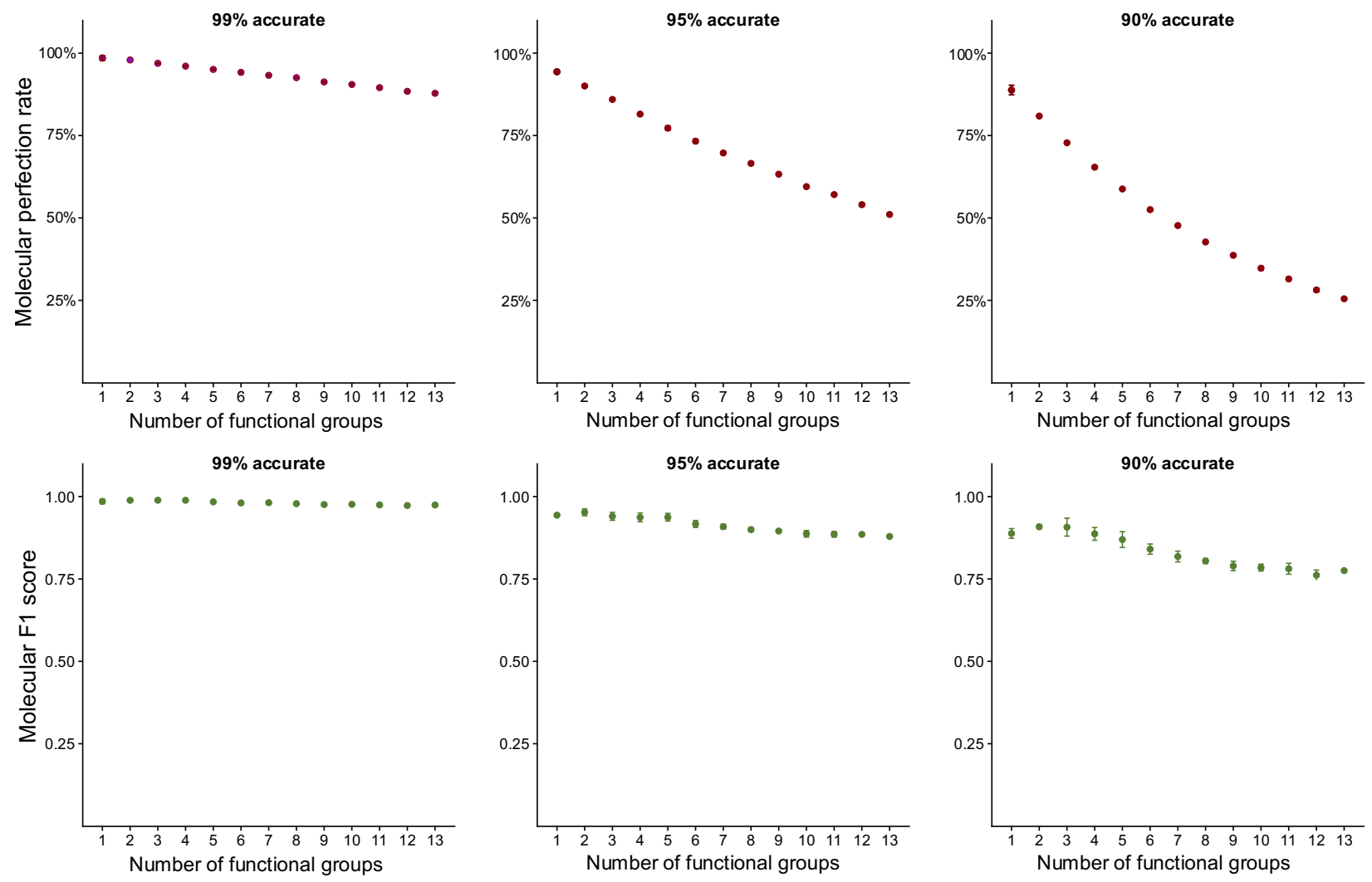
The synthetic models shown in Figure $\mathbf{5} \mathbf{d}$ and Figure $\mathbf{5} \mathbf{d}$ are created to evaluate how molecular perfection rate varies with the introduction of additional functional groups to a machine learning model. Individual synthetic models with differing numbers of functional groups (X-axis) are created, and the corresponding molecular perfection rates are calculated for each synthetic model. Since the purpose of a synthetic model is to serve as a comparison to a machine learning model, the accuracies for each synthetic model is the average of a subset of functional group accuracies taken from the comparison model. These functional group subsets are selected at random every time a synthetic model is created. For example, consider a machine learning model with functional group accuracies of $90 \%, 80 \%$, and $70 \%$; this allows for three synthetic models with two functional groups to be created with accuracies of $85 \%, 80 \%$, and $75 \%$ and a single synthetic model with three functional groups to be created with an accuracy of $80 \%$. For all figures produced in this work, 10 synthetic models are created for each number of functional groups considered (ticks on the $\mathrm{X}$-axis), and the average molecular perfection rate of these 10 models are plotted on the $\mathrm{Y}$ axis.

\section{Details of neural networks}

The final optimization parameters for the IR+MS model:

\begin{tabular}{|ll|}
\hline Layer size & Dropout \\
\hline 237 & 0.457866692938781 \\
\hline 170 & 0.26437107014663824 \\
\hline
\end{tabular}

Batch size: 178.0

For the IR model:

\begin{tabular}{|ll|}
\hline Layer size & Dropout \\
\hline 240 & 0.3820803111613069 \\
\hline 200 & 0.38822353533309584 \\
\hline 131 & 0.008815281710900874 \\
\hline
\end{tabular}

Batch size: 153.0 


\section{Supporting Figures}

a

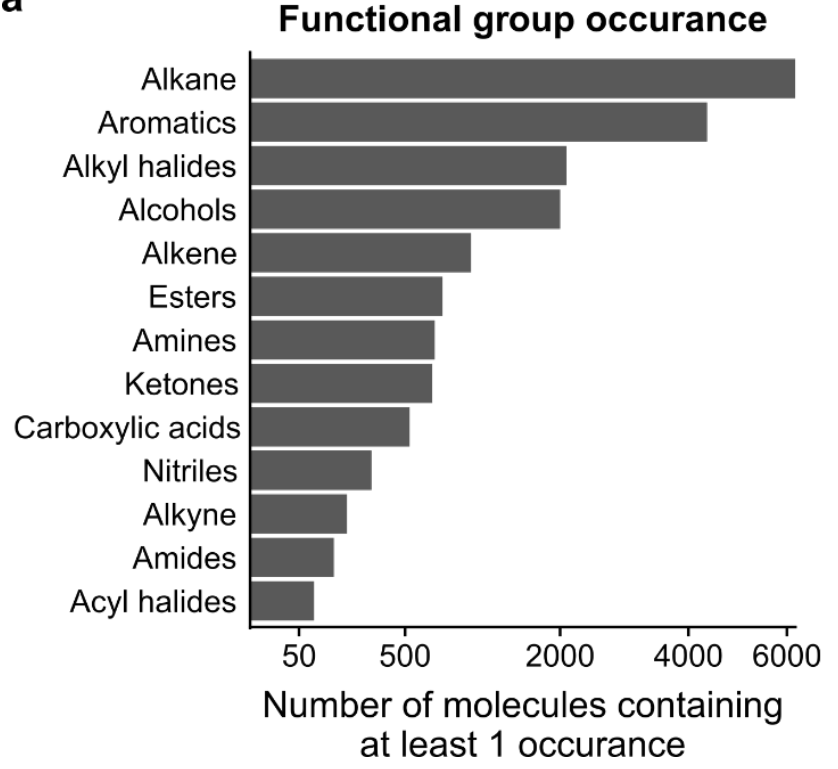

b Distribution of molecular weights

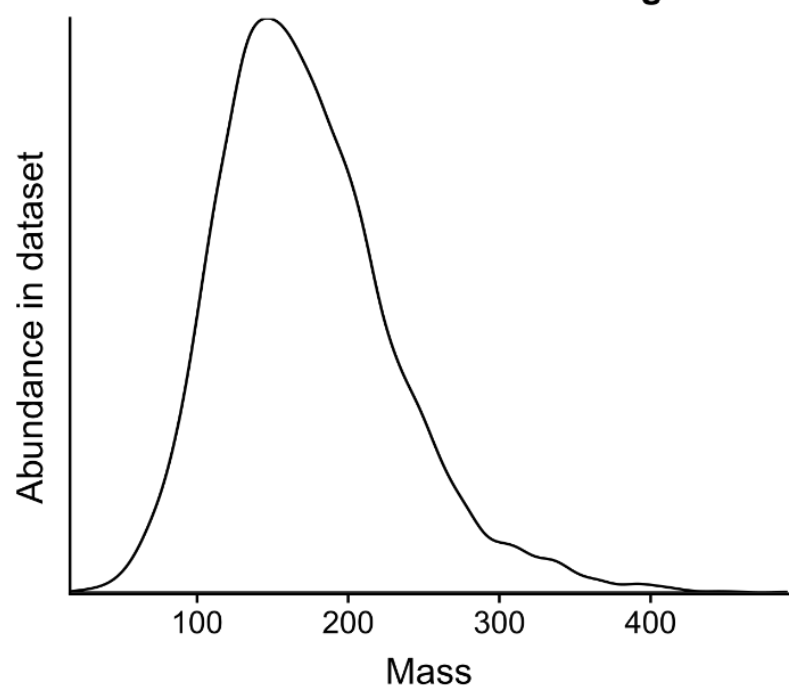

Figure S1: (a) The distribution of various functional groups in the NIST database. (b) The distribution of molecular masses present in the NIST database. 


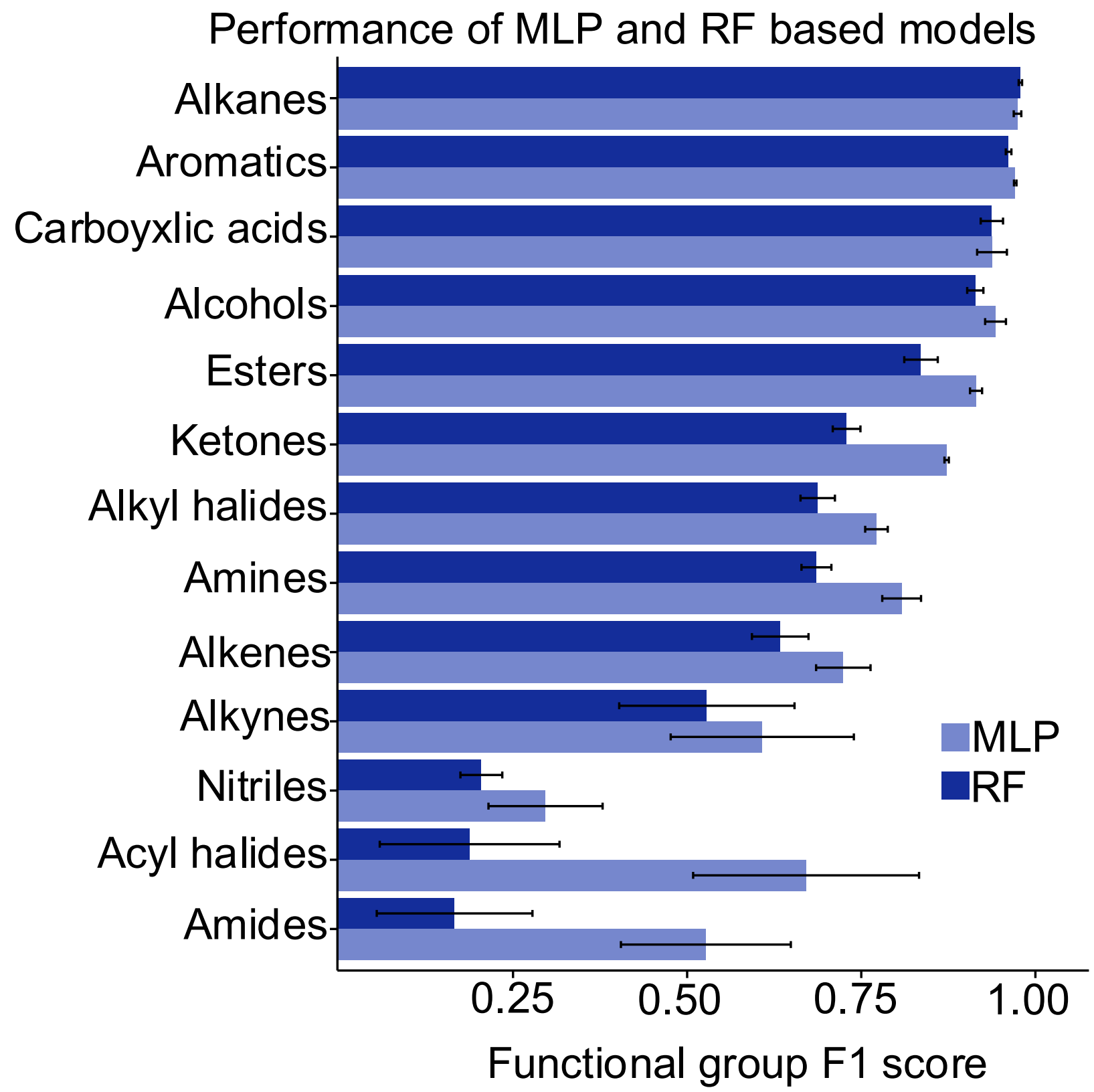

Figure S2: The comparison of Random Forest and Multi-Layered Perception validation set performance for the selected functional groups indicates that the MLP methodology outperforms RF for the majority of functional groups. Both methods were trained on the IR spectra only and no hyperparameters were used to optimize the model. Each bar represents the mean of a 5-fold cross-validation, and the error bars indicate the standard deviation over the 5-folds. Here, the MLP model outperforms random forest and this is apparent for amides, acyl halides, amines, alkyl halides, ketones, and esters. 
a

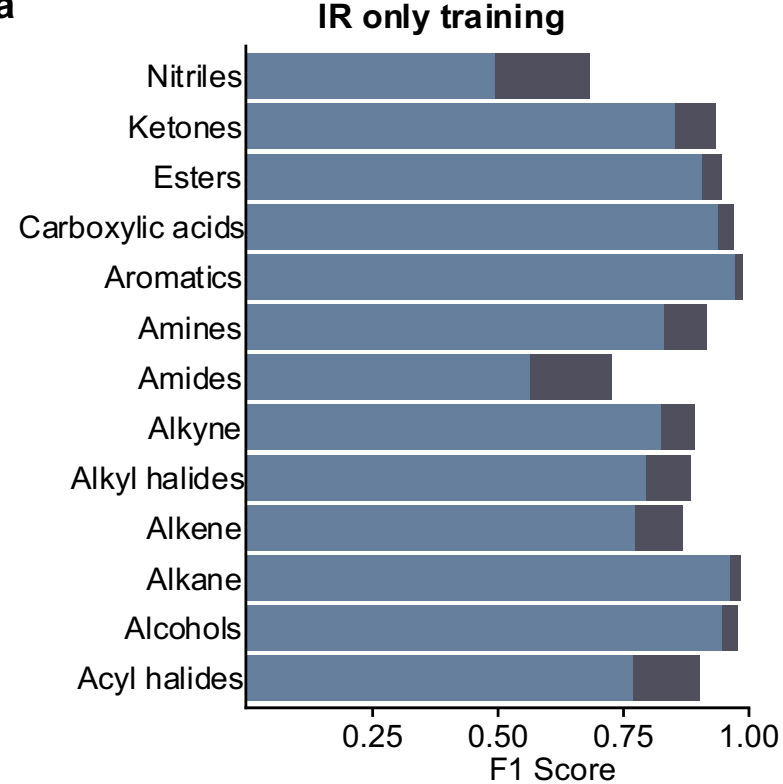

C

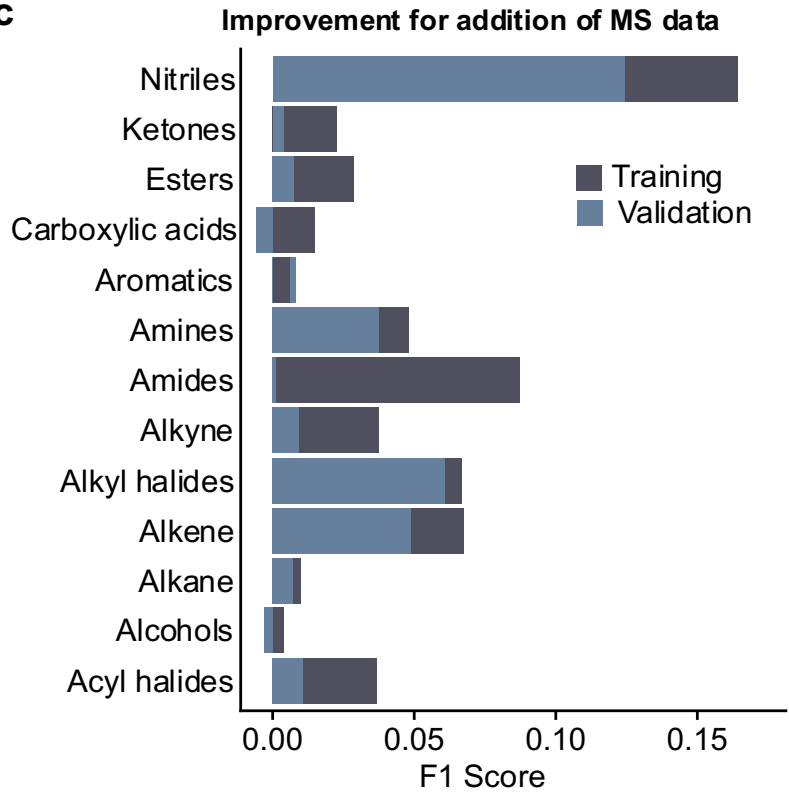

b

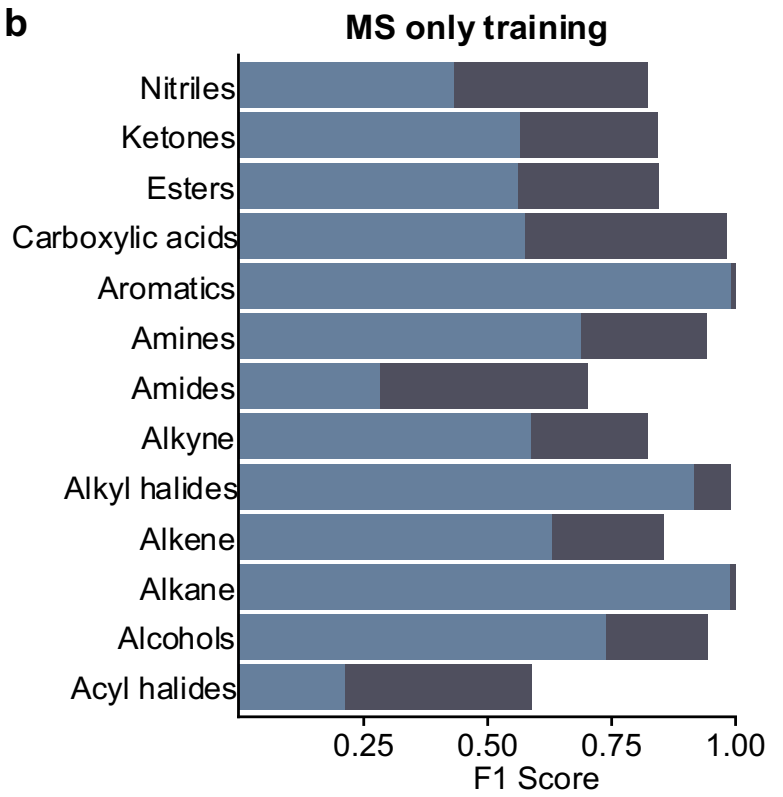

d

IR+MS model

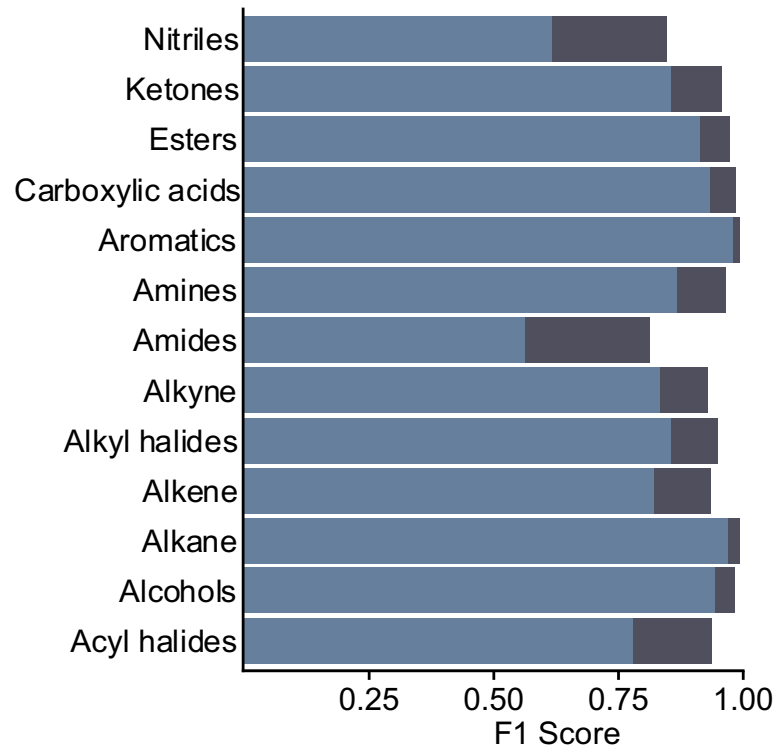

Figure S3: (a) Per functional group F1 for an MLP model trained only on IR data shows that many functional groups can be predicted accurately using an IR only model. (b) Per functional group performance for an MLP model trained only on MS data shows that the model trained only IR data outperforms the model trained only on MS data during K-Fold validation. Also, the MS only model tends to become overtrained in comparison to the IR model potentially due to a greater degree of generalization for IR data. (c) The improvement in performance for each functional group when MS spectra are introduced in addition to IR data. (d) The performance of the combined IR and MS model as measured by functional group F1 score for all functional groups. 

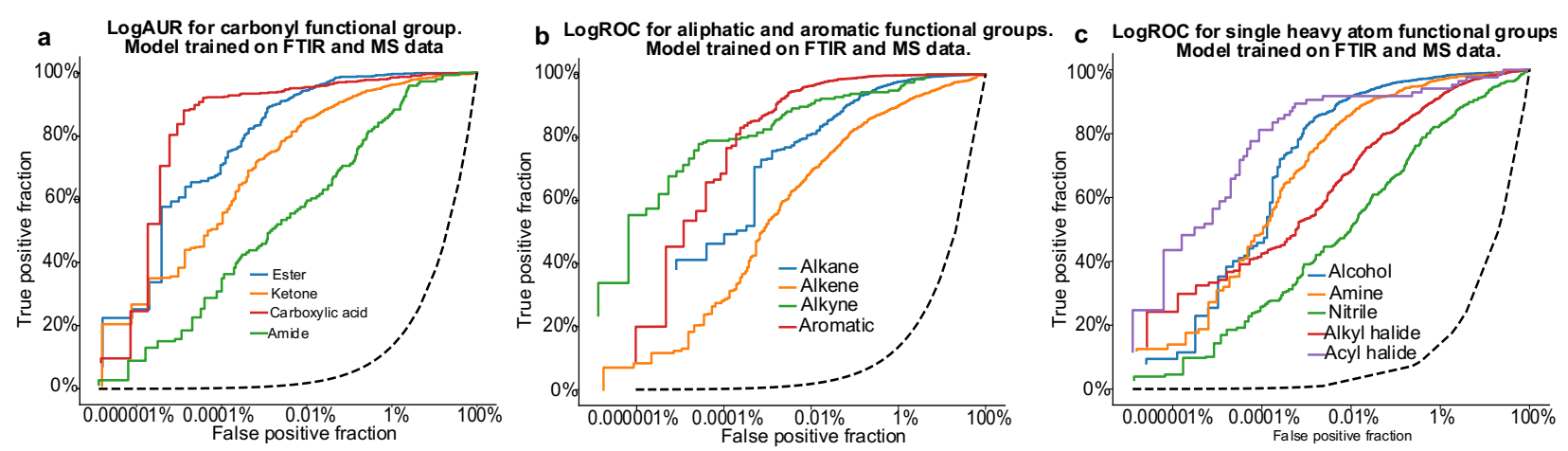

Figure S4: Receiver Operator Characteristic (ROC) plots for the model trained on both IR and MS spectra. (a) performance for carbonyl functional groups, (b) groups consisting of only carbon and hydrogen, and (c)the remaining functional groups. The underperformance of amides and nitriles can be discerned from these plots. These plots also allow us to select the best threshold value for each functional group which maximizes the F-1 score for that functional group. 
Functional group F1 score before and after addition of new groups IR only training

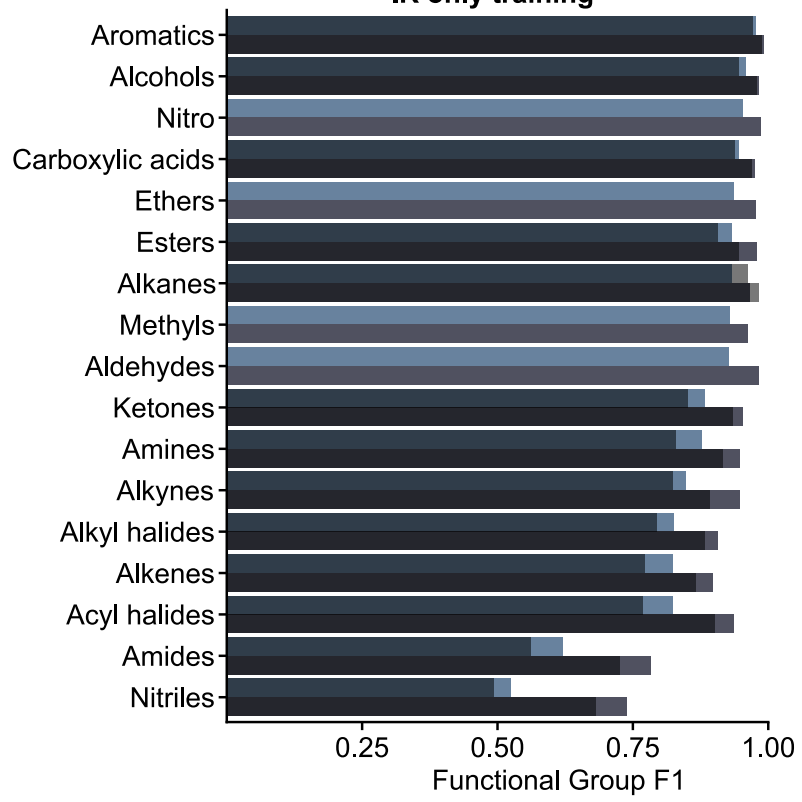

b

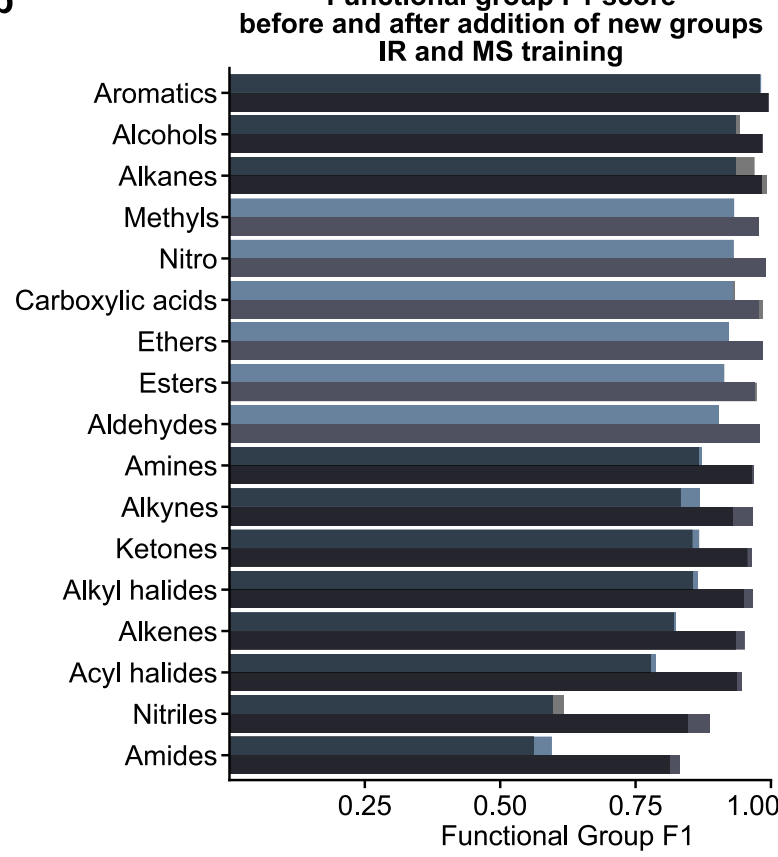

Training set $\square$ Validation set $\square$ Performance of the original model $\square$ New functional group $\square$ Redefined group

C

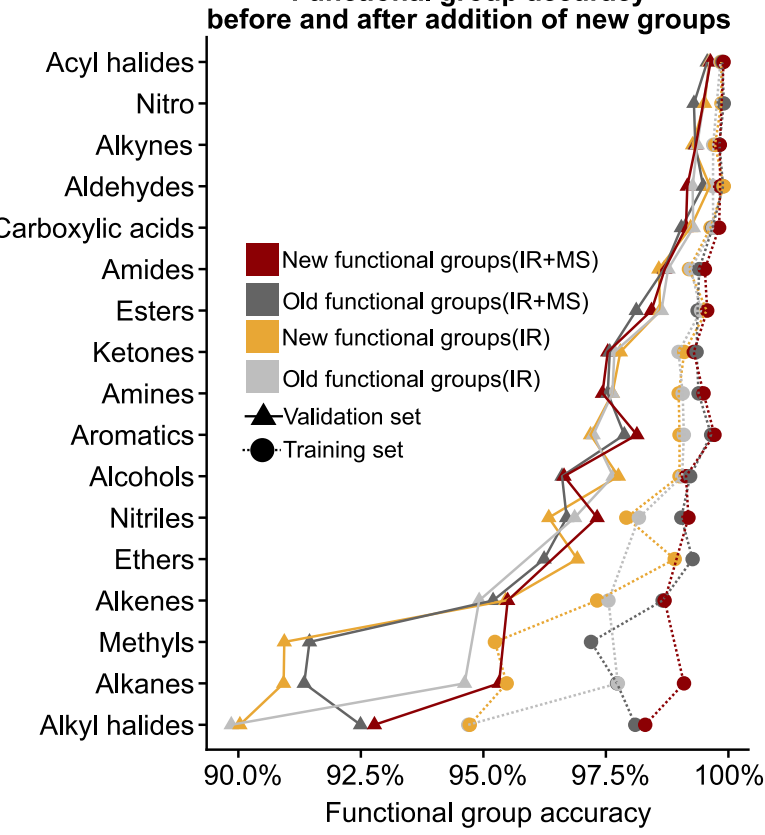

d

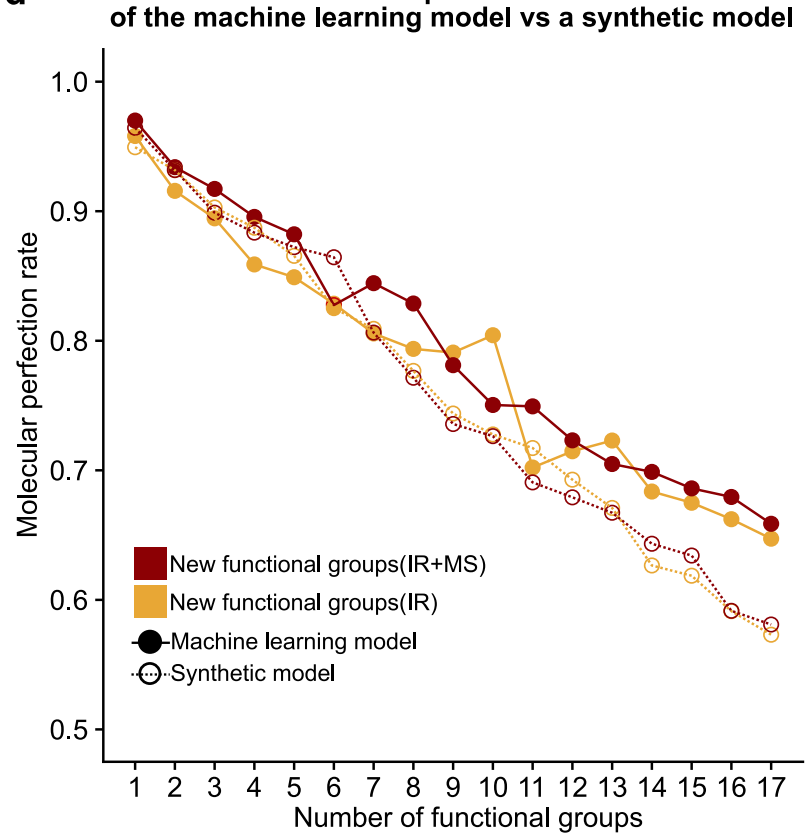

Figure S5: A extended version of Figure 5 in the main text. Here, we show both training and validation set results for panels a-c in the original figure. 


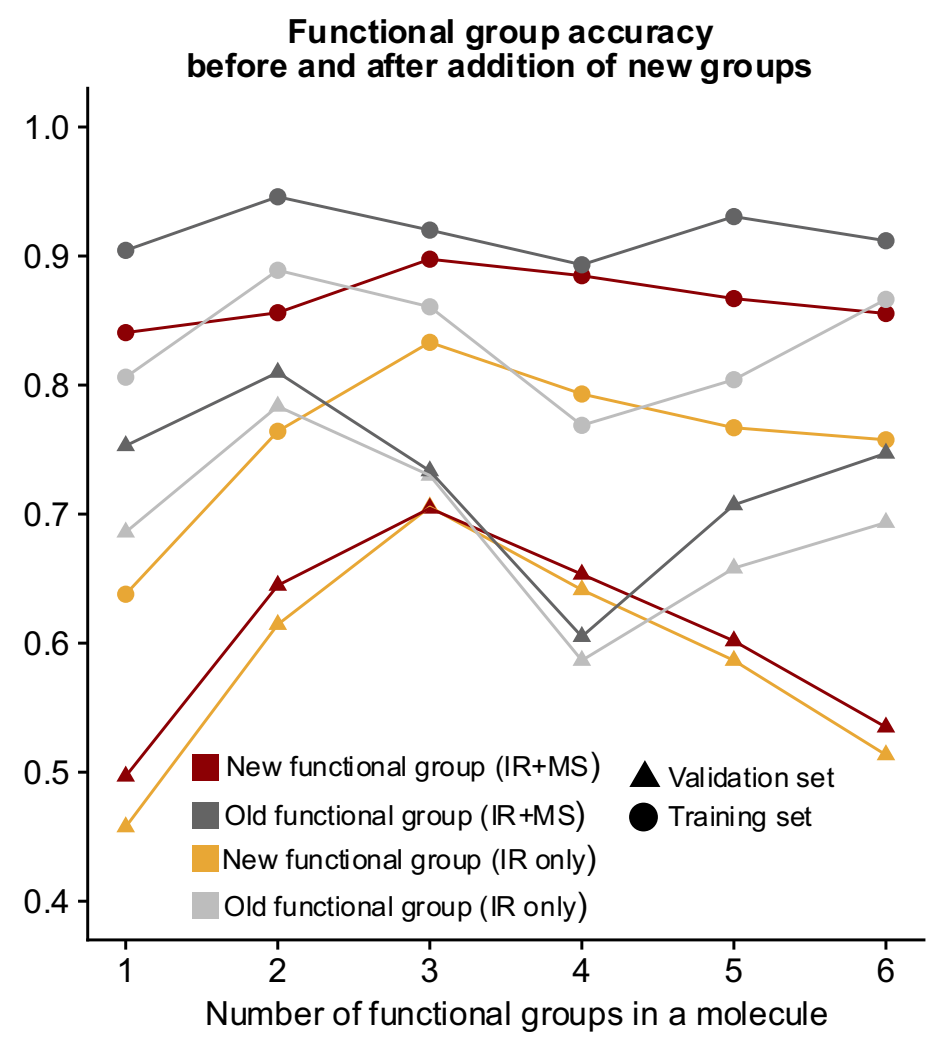

Figure $\mathbf{S 6}$ The molecular perfection rate calculated on molecules with a specific number of functional groups for both the original and new set of functional groups. 


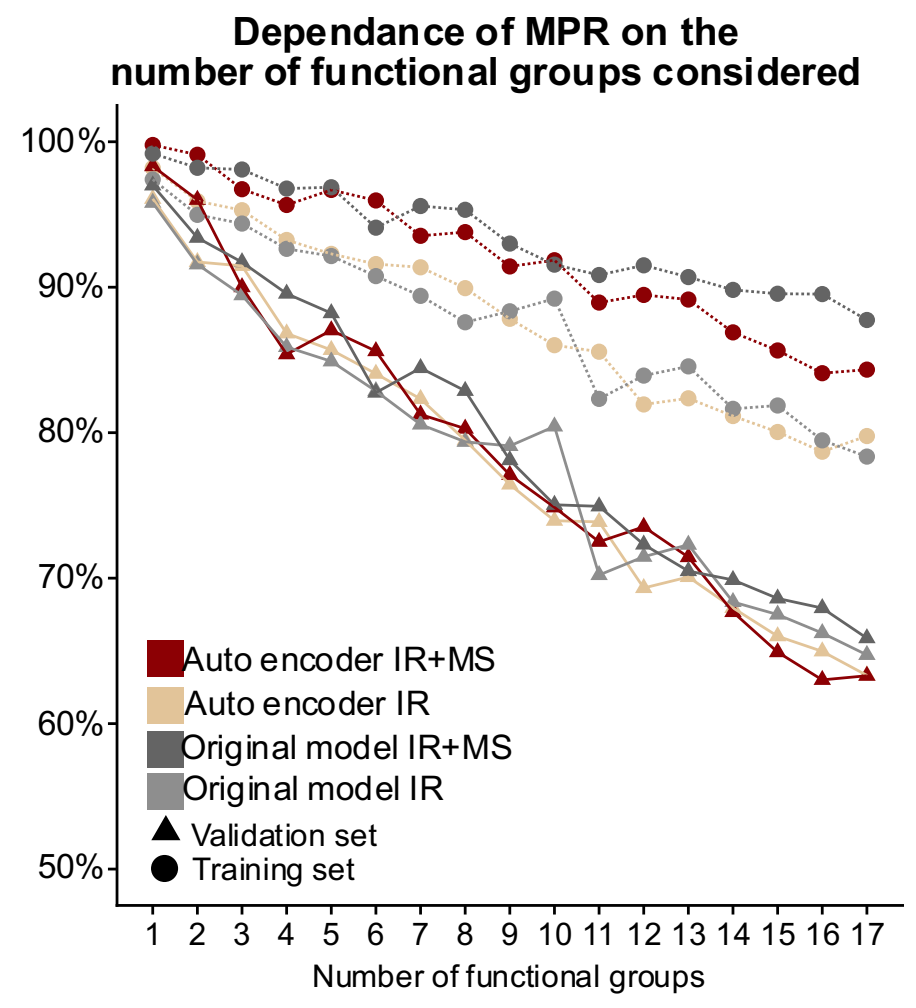

Figure S7: A version of Figure $\mathbf{5} \mathbf{d}$ which includes both the training set data and the autoencoder model. This figure shows that the autoencoder and training set data follow the same trend as the one found in the original model's validation set. 


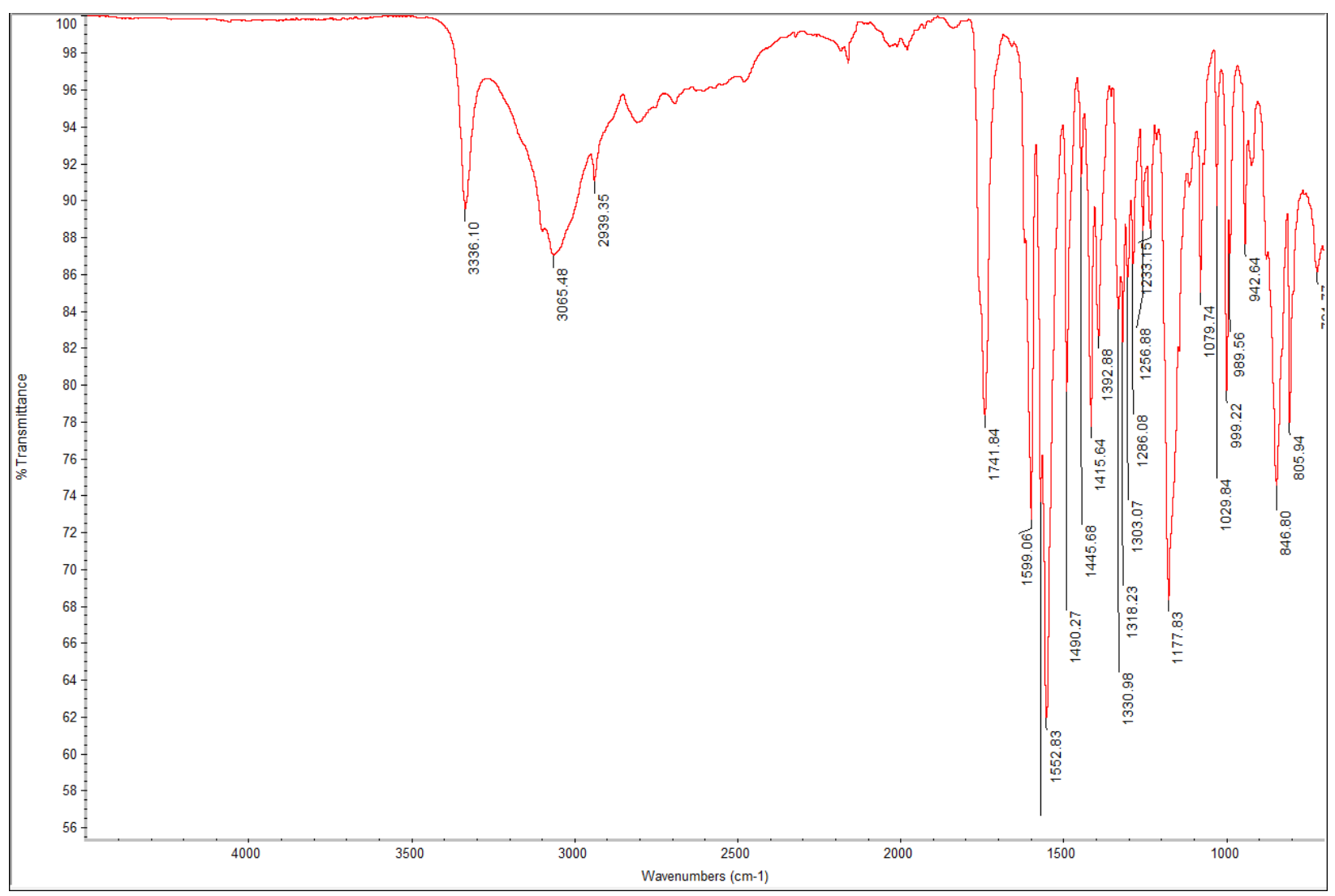

Figure S8: Recorded ATR-IR spectrum for mixture 1. 


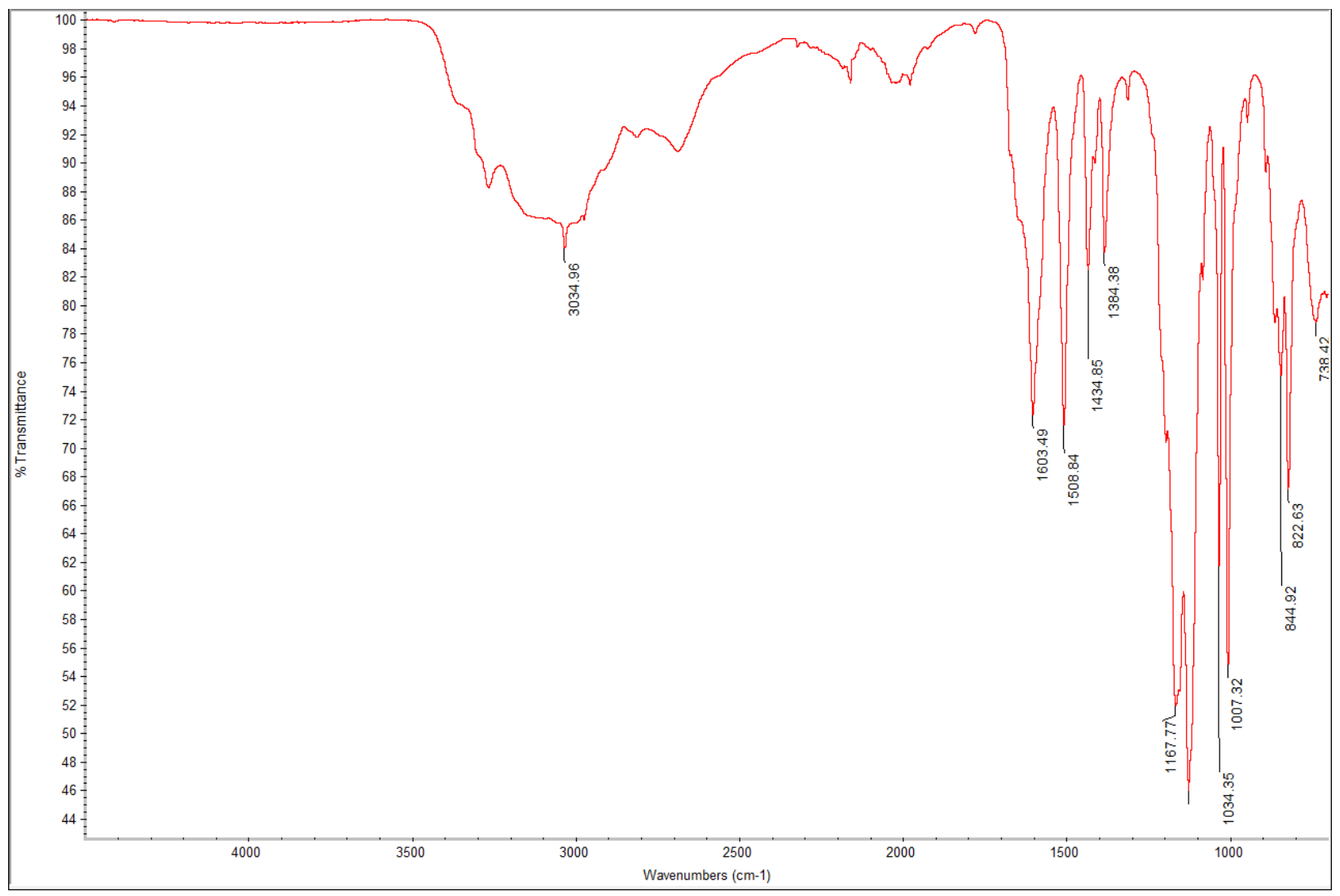

Figure S9: Recorded ATR-IR spectrum for mixture 2. 


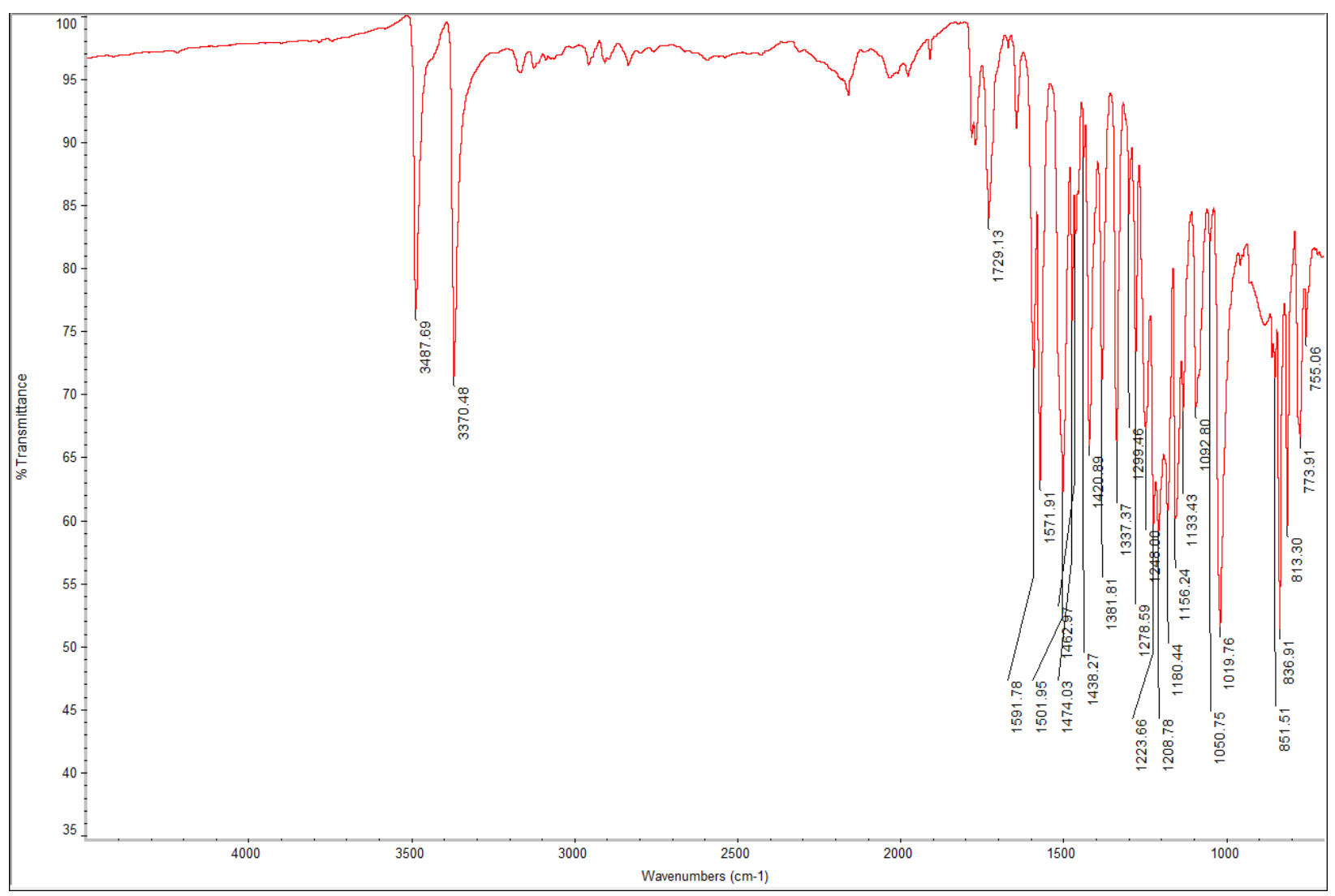

Figure S10: Recorded ATR-IR spectrum for mixture 3. 
Supporting Tables

\begin{tabular}{|l|l|l|l|l|l|}
\hline Functional Group & Fold1 & Fold2 & Fold3 & Fold4 & Fold5 \\
\hline Alkane & 0.979032 & 0.976801 & 0.975889 & 0.978278 & 0.982206 \\
\hline Alkene & 0.648829 & 0.616949 & 0.578778 & 0.634441 & 0.689139 \\
\hline Alkyne & 0.367347 & 0.629630 & 0.509804 & 0.458333 & 0.676471 \\
\hline Alcohols & 0.902405 & 0.900000 & 0.919127 & 0.922293 & 0.924731 \\
\hline Amines & 0.647303 & 0.695652 & 0.692982 & 0.695652 & 0.696517 \\
\hline Nitriles & 0.246154 & 0.222222 & 0.181818 & 0.200000 & 0.172414 \\
\hline Aromatics & 0.957637 & 0.958546 & 0.959868 & 0.965123 & 0.966496 \\
\hline Alkyl halides & 0.679083 & 0.701001 & 0.723837 & 0.665765 & 0.667638 \\
\hline Esters & 0.805556 & 0.842105 & 0.860215 & 0.816000 & 0.855072 \\
\hline Ketones & 0.757991 & 0.720000 & 0.736364 & 0.725664 & 0.704663 \\
\hline Carboxylic acids & 0.931217 & 0.944724 & 0.928230 & 0.921569 & 0.961905 \\
\hline Acyl halides & 0.222222 & 0.105263 & 0.100000 & 0.400000 & 0.111111 \\
\hline Amides & 0.050000 & 0.258065 & 0.071429 & 0.303003 & 0.148148 \\
\hline
\end{tabular}

Table S1: Functional group F-1 scores for the random forest model.

\begin{tabular}{|l|l|l|l|l|l|}
\hline Functional Group & Fold1 & Fold2 & Fold3 & Fold4 & Fold5 \\
\hline Alkanes & 0.969475 & 0.979757 & 0.970094 & 0.971729 & 0.980640 \\
\hline Alkenes & 0.689459 & 0.728291 & 0.706849 & 0.706849 & 0.789644 \\
\hline Alkynes & 0.423077 & 0.678571 & 0.677966 & 0.520000 & 0.739726 \\
\hline Alcohols & 0.923497 & 0.932015 & 0.955446 & 0.944649 & 0.958333 \\
\hline Amines & 0.788530 & 0.776371 & 0.847826 & 0.807407 & 0.819672 \\
\hline Nitriles & 0.238806 & 0.243243 & 0.289157 & 0.273973 & 0.438356 \\
\hline Aromatics & 0.974011 & 0.969907 & 0.969461 & 0.971098 & 0.970862 \\
\hline Alkyl halides & 0.768229 & 0.749672 & 0.786842 & 0.788698 & 0.765743 \\
\hline Esters & 0.926380 & 0.916944 & 0.903427 & 0.909747 & 0.917492 \\
\hline Ketones & 0.876404 & 0.874172 & 0.868526 & 0.873563 & 0.870293 \\
\hline Carboxylic acids & 0.953846 & 0.920000 & 0.939535 & 0.912621 & 0.962617 \\
\hline Acyl halides & 0.416667 & 0.714286 & 0.689655 & 0.666667 & 0.866667 \\
\hline Amides & 0.333333 & 0.600000 & 0.550000 & 0.651163 & 0.500000 \\
\hline
\end{tabular}

Table S2: Functional group F-1 scores for the initial neural network model. 


\begin{tabular}{|l|r|r|}
\hline Functional Group & Training set F1 & Validation set F1 \\
\hline Alkane & 0.983057 & 0.962597 \\
\hline Alkene & 0.866956 & 0.771962 \\
\hline Alkyne & 0.891495 & 0.824410 \\
\hline Alcohols & 0.978567 & 0.946291 \\
\hline Amines & 0.916645 & 0.829724 \\
\hline Nitriles & 0.682049 & 0.493131 \\
\hline Aromatics & 0.987723 & 0.971455 \\
\hline Alkyl halides & 0.883381 & 0.794842 \\
\hline Esters & 0.945287 & 0.906326 \\
\hline Ketones & 0.933960 & 0.851401 \\
\hline Carboxylic acids & 0.970379 & 0.938528 \\
\hline Acyl halides & 0.901172 & 0.767982 \\
\hline Amides & 0.726499 & 0.562125 \\
\hline
\end{tabular}

Table S3: Functional group F-1 scores for the neural network model trained on only IR spectra

\begin{tabular}{|l|r|r|}
\hline fg & Training set F1 & Validation set F1 \\
\hline Alkane & 0.999203 & 0.988438 \\
\hline Alkene & 0.854542 & 0.629076 \\
\hline Alkyne & 0.823007 & 0.586774 \\
\hline Alcohols & 0.944003 & 0.738918 \\
\hline Amines & 0.940923 & 0.686877 \\
\hline Nitriles & 0.821942 & 0.431075 \\
\hline Aromatics & 0.999599 & 0.990165 \\
\hline Alkyl halides & 0.990067 & 0.916458 \\
\hline Esters & 0.844197 & 0.561388 \\
\hline Ketones & 0.843226 & 0.565298 \\
\hline Carboxylic acids & 0.981144 & 0.574958 \\
\hline Acyl halides & 0.589059 & 0.210796 \\
\hline Amides & 0.701243 & 0.282036 \\
\hline
\end{tabular}

Table S4: Functional group F-1 scores for the neural network model trained on only MS spectra 


\begin{tabular}{|l|r|r|}
\hline Functional Group & Training set F1 & Validation set F1 \\
\hline Alkane & 0.992912 & 0.969812 \\
\hline Alkene & 0.934595 & 0.820889 \\
\hline Alkyne & 0.928958 & 0.833759 \\
\hline Alcohols & 0.982569 & 0.943450 \\
\hline Amines & 0.964915 & 0.867276 \\
\hline Nitriles & 0.846275 & 0.617405 \\
\hline Aromatics & 0.993621 & 0.979649 \\
\hline Alkyl halides & 0.950192 & 0.855821 \\
\hline Esters & 0.974097 & 0.913863 \\
\hline Ketones & 0.956765 & 0.855171 \\
\hline Carboxylic acids & 0.985346 & 0.932786 \\
\hline Acyl halides & 0.938061 & 0.778668 \\
\hline Amides & 0.813684 & 0.563190 \\
\hline
\end{tabular}

Table S5: Functional group F-1 scores for the neural network model trained on both IR and MS spectra

\begin{tabular}{|l|l|l|l|l|l|}
\hline fg & Fold1 & Fold2 & Fold3 & Fold4 & Fold5 \\
\hline Alkane & 0.975453 & 0.97485 & 0.977956 & 0.984628 & 0.971593 \\
\hline Alkene & 0.820779 & 0.796657 & 0.813648 & 0.782396 & 0.833333 \\
\hline Alkyne & 0.818182 & 0.852941 & 0.857143 & 0.900000 & 0.707071 \\
\hline Alcohols & 0.951654 & 0.931789 & 0.932515 & 0.931436 & 0.929397 \\
\hline Amines & 0.879433 & 0.879433 & 0.879699 & 0.853047 & 0.861314 \\
\hline Nitriles & 0.647059 & 0.715596 & 0.533333 & 0.637931 & 0.632653 \\
\hline Aromatics & 0.979167 & 0.982739 & 0.978748 & 0.984438 & 0.982416 \\
\hline Alkyl halides & 0.871338 & 0.849449 & 0.878107 & 0.881748 & 0.875931 \\
\hline Esters & 0.929487 & 0.900000 & 0.914286 & 0.924528 & 0.923588 \\
\hline Ketones & 0.853755 & 0.892308 & 0.817814 & 0.881890 & 0.828571 \\
\hline Carboxylic acids & 0.92093 & 0.900990 & 0.955665 & 0.940594 & 0.897561 \\
\hline Acyl halides & 0.785714 & 0.857143 & 0.833333 & 0.838710 & 0.709677 \\
\hline Amides & 0.612245 & 0.640000 & 0.478261 & 0.612245 & 0.690909 \\
\hline
\end{tabular}

Table S6: Functional group F-1 scores for single neural networks trained on both IR and MS spectra 
Table S7: MPR and MF1 values for a multitask model trained on only IR spectra

\begin{tabular}{|l|r|r|}
\hline & Molecular Perfection Rate & Molecular F-1 \\
\hline Training set & $92.5571 \%$ & 0.982041 \\
\hline Validation set & $74.9085 \%$ & 0.931506 \\
\hline
\end{tabular}

Table S8: MPR and MF1 values for a multitask model trained on IR and MS spectra 


\begin{tabular}{|l|r|r|}
\hline Functional Group & Training set F1 & Validation set F1 \\
\hline Alkanes & 0.966563 & 0.932969 \\
\hline Alkenes & 0.898341 & 0.823709 \\
\hline Alkynes & 0.946598 & 0.847545 \\
\hline Alcohols & 0.981538 & 0.957765 \\
\hline Amines & 0.948083 & 0.877436 \\
\hline Aitriles & 0.739183 & 0.525128 \\
\hline Aromatics & 0.991503 & 0.976025 \\
\hline Alkyl halides & 0.907264 & 0.825761 \\
\hline Esters & 0.978914 & 0.933366 \\
\hline Ketones & 0.952114 & 0.882585 \\
\hline Aldehydes & 0.982074 & 0.927797 \\
\hline Carboxylic acids & 0.974353 & 0.944752 \\
\hline Acyl halides & 0.936764 & 0.822867 \\
\hline Amides & 0.783791 & 0.620740 \\
\hline Methyl & 0.962598 & 0.928545 \\
\hline Ether & 0.977310 & 0.935875 \\
\hline Nitro & 0.986419 & 0.953173 \\
\hline
\end{tabular}

Table S9: Functional group F-1 scores for a model trained on only IR with the new definitions of functional groups

\begin{tabular}{|l|r|r|}
\hline Functional Group & Training set F1 & Validation set F1 \\
\hline Alkane & 0.983110 & 0.935748 \\
\hline Alkene & 0.951914 & 0.825343 \\
\hline Alkyne & 0.966157 & 0.869274 \\
\hline Alcohols & 0.985552 & 0.935951 \\
\hline Amines & 0.969121 & 0.873207 \\
\hline Nitriles & 0.887506 & 0.598101 \\
\hline Aromatics & 0.997007 & 0.981913 \\
\hline Alkyl halides & 0.966182 & 0.865727 \\
\hline Esters & 0.970721 & 0.912860 \\
\hline Ketones & 0.965129 & 0.867477 \\
\hline Aldehydes & 0.979790 & 0.903850 \\
\hline Carboxylic acids & 0.977540 & 0.930756 \\
\hline Acyl halides & 0.945896 & 0.788083 \\
\hline Amides & 0.832065 & 0.595560 \\
\hline Methyls & 0.977781 & 0.932062 \\
\hline Ethers & 0.984980 & 0.923053 \\
\hline Nitros & 0.990951 & 0.931536 \\
\hline
\end{tabular}

Table S10: Functional group F-1 scores for a model trained on IR and MS spectra with the new definitions of functional groups 


\begin{tabular}{|l|r|r|}
\hline & Molecular Perfection Rate & Molecular F-1 \\
\hline Training set & $79.1323 \%$ & 0.955077 \\
\hline Validation set & $64.0335 \%$ & 0.909212 \\
\hline
\end{tabular}

Table S11: MPR and MF1 values for a multitask model trained on only IR spectra with the new definitions of functional groups

\begin{tabular}{|l|r|r|}
\hline & Molecular Perfection Rate & Molecular F-1 \\
\hline Training set & $87.8871 \%$ & 0.975642 \\
\hline Validation set & $65.2510 \%$ & 0.912017 \\
\hline
\end{tabular}

Table S12: MPR and MF1 values for a multitask model trained on IR and MS spectra 


\begin{tabular}{|l|r|r|}
\hline Functional Group & Training set F1 & Validation set F1 \\
\hline Alkane & 0.968777 & 0.93169 \\
\hline Alkene & 0.907346 & 0.812864 \\
\hline Alkyne & 0.945042 & 0.851205 \\
\hline Alcohols & 0.97892 & 0.944236 \\
\hline Amines & 0.946405 & 0.852841 \\
\hline Nitriles & 0.717182 & 0.488428 \\
\hline Aromatics & 0.992644 & 0.974879 \\
\hline Alkyl halides & 0.907742 & 0.810426 \\
\hline Esters & 0.979923 & 0.922709 \\
\hline Ketones & 0.951888 & 0.867387 \\
\hline Aldehydes & 0.976048 & 0.918015 \\
\hline Carboxylic acids & 0.971139 & 0.941297 \\
\hline Acyl halides & 0.920298 & 0.791876 \\
\hline Amides & 0.788451 & 0.597016 \\
\hline Methysl & 0.963815 & 0.932059 \\
\hline Ethers & 0.973455 & 0.923417 \\
\hline Nitros & 0.98336 & 0.946973 \\
\hline
\end{tabular}

Table S13: Functional group F-1 scores for a model trained using an autoencoder on only IR with the new definitions of functional groups

\begin{tabular}{|l|r|r|}
\hline Functional Group & Training set F1 & Validation set F1 \\
\hline Alkane & 0.984136 & 0.932257 \\
\hline Alkene & 0.947195 & 0.819603 \\
\hline Alkyne & 0.958650 & 0.848086 \\
\hline Alcohols & 0.978334 & 0.910960 \\
\hline Amines & 0.960173 & 0.852991 \\
\hline Nitriles & 0.854644 & 0.553305 \\
\hline Aromatics & 0.996893 & 0.982649 \\
\hline Alkyl halides & 0.963728 & 0.855594 \\
\hline Esters & 0.969606 & 0.913754 \\
\hline Ketones & 0.964384 & 0.857152 \\
\hline Aldehydes & 0.979850 & 0.866663 \\
\hline Carboxylic acids & 0.979510 & 0.917079 \\
\hline Acyl halides & 0.952464 & 0.736802 \\
\hline Amides & 0.844232 & 0.557778 \\
\hline Methyls & 0.978014 & 0.930977 \\
\hline Ethers & 0.980859 & 0.919104 \\
\hline Nitros & 0.987188 & 0.933380 \\
\hline
\end{tabular}

Table S14: Functional group F-1 scores for a model trained using an autoencoder on IR and MS with the new definitions of functional groups 


\begin{tabular}{|l|r|r|}
\hline & Molecular Perfection Rate & Molecular F-1 \\
\hline Training set & $78.8955 \%$ & 0.955907 \\
\hline Validation set & $62.5593 \%$ & 0.904820 \\
\hline
\end{tabular}

Table S15: MPR and MF1 values for a model trained using an autoencoder on only IR and with the new definitions of functional groups

\begin{tabular}{|l|r|r|}
\hline & Molecular Perfection Rate & Molecular F-1 \\
\hline Training set & $86.8895 \%$ & 0.973454 \\
\hline Validation set & $62.5726 \%$ & 0.905013 \\
\hline
\end{tabular}

Table S16: MPR and MF1 values for a model trained using an autoencoder on IR and MS and with the new definitions of functional groups 
References

[1] S. Kim, P. A. Thiessen, E. E. Bolton, J. Chen, G. Fu, A. Gindulyte, L. Han, J. He, S. He, B. A. Shoemaker, et al., Nucleic Acids Res. 2016, 44, DOI 10.1093/nar/gkv951.

[2] G. A. Landrum, n.d.

[3] DayLight, n.d.

[4] R. Nalla, R. Pinge, M. Narwaria, B. Chaudhury, in Proc. ACM India Jt. Int. Conf. Data Sci. Manag. Data-CoDS-COMAD '18, 2018, pp. 201-209.

[5] R. J. Fessenden, L. Györgyi, J. Chem. Soc., Perkin Trans. 2 1991, 1755-1762.

[6] E. W. Robb, M. E. Munk, Mikrochim. Acta 1990, 100, 131-155.

[7] F. Chollet, Keras, 2015.

[8] J. Gabel, J. Desaphy, D. Rognan, J. Chem. Inf. Model. 2014, 54, 2807-2815.

[9] J. T. Springenberg, A. Dosovitskiy, T. Brox, M. Riedmiller, 2014.

[10] M. D. Zeiler, R. Fergus, in Lect. Notes Comput. Sci. (Including Subser. Lect. Notes Artif. Intell. Lect. Notes Bioinformatics), 2014, pp. 818-833. 


\section{Spectral deep learning for prediction and prospective validation of functional groups}

Jonathan A. Fine ${ }^{1,{ }^{\dagger}}$, Anand A. Rajasekar ${ }^{2,{ }^{\dagger}}$, Krupal P. Jethava ${ }^{1}$, Gaurav Chopra ${ }^{1}$ *

[1] Department of Chemistry

Purdue University

560 Oval Drive, West Lafayette, IN 47907

[2] Department of Biological Engineering

Bhupat and Jyoti Mehta School of Biosciences

Indian Institute of Technology Madras, Chennai 600036, India

$\left.{ }^{[}{ }^{\dagger}\right]$ These authors contributed equally to this work. 


\begin{abstract}
State-of-the-art identification of the functional groups present in an unknown chemical entity requires expertise of a skilled spectroscopist to analyse and interpret Fourier Transform InfraRed (FTIR), Mass Spectroscopy (MS) and/or Nuclear Magnetic Resonance (NMR) data. This process can be time-consuming and error-prone, especially for complex chemical entities that poorly characterized in the literature, or inefficient to use with synthetic robots producing molecules at an accelerated rate. Herein, we introduce a fast, multi-label deep neural network for accurately identifying all the functional groups of unknown compounds using a combination of FTIR and MS spectra. We do not use any database, pre-established rules, procedures, or peakmatching methods. Our trained neural network reveals patterns typically used by human chemists to identify standard groups. Finally, we experimentally validated our neural network, trained on single compounds, to predict functional groups in compound mixtures. Our methodology showcases practical utility for future use in autonomous analytical detection.
\end{abstract}




\section{Introduction}

The arrangement of atoms within a molecule dictates its physical, chemical, and spectral properties. Small discrete, or large repeating arrangements of atoms which give rise to measurable changes in a molecule's reactivity ${ }^{1-3}$, boiling point ${ }^{4,5}$, melting point ${ }^{6,7}$, and other characteristics are called functional groups. Given the structural formula of a molecule, a chemist can identify functional groups present (e.g. aldehyde, carboxylic acid, alcohol, etc.) and can postulate characteristic reactivity and physical properties for a given molecule based on the presence of these groups. Therefore, the identification of functional groups present within an unknown compound is a key step in qualitative organic synthesis and structure elucidation; it is routinely practiced by chemists to validate the synthesis of novel small molecules or identify unknown structures in complex mixtures. Techniques for assigning functional groups based on 'rules of thumb' or by matching profiles from known databases are commonly applied in organic chemistry $^{8}$, metabolomics ${ }^{9,10}$, and forensic sciences ${ }^{11-13}$. Furthermore, monitoring of functional group changes can be used to determine the progress of a reaction, ${ }^{14}$ and can even be used to identify the components of complex mixtures for a reaction coordinate.

Chemists often rely on spectroscopic techniques like FTIR, MS, and NMR for the assignment of functional groups. FTIR utilizes the frequencies associated with the bonds in a molecule, which typically vibrate around $4000 \mathrm{~cm}^{-1}$ to $400 \mathrm{~cm}^{-1}$, known as the Infrared region of the electromagnetic spectrum ${ }^{8}$. This region is associated with specific frequencies that change the oscillating patterns of chemical bonds in the analyte, resulting in an IR spectrum ${ }^{15}$. Typically, a spectroscopist manually analyses this spectrum to identify patterns corresponding to a given functional group using previously established rules and principals ${ }^{8}$, a time-consuming process subject to human bias and interpretation. Alternatively, if the compound has previously been characterized, the spectroscopist can use software to match the peaks of the analyte to a database of known compounds for identification ${ }^{16}$.

Mass spectroscopy (MS) is another technique commonly used by chemists for the identification of unknown compounds ${ }^{8}$. One of the first, and still a popular MS ionization technique is electron ionization $(\mathrm{EI}-\mathrm{MS})^{17}$, a method performed by bombarding the analyte in the gas phase with high energy electrons $(\sim 70 \mathrm{eV})$ for molecular ionization. The resulting cationic radicals are 
energetically unstable and break apart, resulting in smaller charged particle fragments that are specific to the analyte. Such fragmentation patterns are dependent on molecular functional groups and their arrangements with other functional groups and motifs. The abundance of fragments with a given mass to charge ratio $(\mathrm{m} / \mathrm{z})$ is recorded and reported as the mass spectrum. These spectra are used to search through a database of MS peaks of known compounds, but large-scale automated identification of unknown molecules is still a major challenge $\mathrm{e}^{9,18-20}$.

Human intervention to analyse FTIR or MS spectrum is useful but to achieve the next generation of autonomous instrumentation for reaction screening, a completely automated method for determining whether a reaction occurred is required. The current approaches to automating functional group identification are similar to those applied by humans, using a set of rules and pattern (peak) matching to map spectra to a functional group ${ }^{19,21}$. Such methods typically utilize only selected spectral regions to identify functional groups, and often afford relatively low confidence predictions owing to a limited database of known compounds ${ }^{18}$. Furthermore, to our knowledge, these methods can only incorporate data from a single spectral technique (i.e., either FTIR or MS) and ignore relationships between different spectral data for identification. Hence, there is a need for automated and accurate methods capable of multiplespectra integration without the use of pre-established patterns on known databases. Such methods will need minimal-to-no human intervention towards the realization of automated synthetic robots that screen functional groups and combine spectral data to validate each step during reaction screening and multi-step automated synthesis ${ }^{22}$. The state-of-the-art robot for automated reaction detection currently employs different techniques to determine the presence of a reaction ${ }^{23}$, but it is limited to identify the presence of pre-defined known compounds. It is a major challenge to develop fully automated robots to discover new reactions that produce unexpected products. Our goal is to extend the capabilities of these automated synthetic robots by developing a fast, automated methodology for functional group determination that can be used in real-time, thereby enabling reaction screening through the identification of functional group changes in a database-free manner.

Machine Learning (ML) is a set of techniques used by computers to perform a specific task without an explicit set of instructions provided by the user. ML techniques have been 
successfully applied to multiple chemical problems in recent years, and still show promise for the advancement of several areas of chemistry. Popular machine learning architectures, such as Random Forest ${ }^{24-26}$, Multiple Layer Perception ${ }^{27-29}$, Generalized Adversarial Networks ${ }^{30-34}$, and Recurrent Neural Networks ${ }^{35-37}$ have been used on chemical data for small-molecule design ${ }^{38,39}$, metabolism ${ }^{40,41}$, toxicology $4^{40,42}$, photo-electric properties, solubility, and retrosynthesis ${ }^{35,37}$. It has been shown that direct molecule as a subgraph of groups of atoms (i.e., functional groups) has distinct advantages over fingerprinting methods ${ }^{43,44}$. The representation of a molecule or dataset can be reduced to a lower-dimensional latent space by using an autoencoder ${ }^{39}$. Here, we also used an encoder to create a corresponding latent space based on spectra to predict functional groups which may also be useful to design molecules for specific spectral properties. A few ML techniques to analyse spectra has been used previously ${ }^{45-49}$ but such attempts for function group prediction used only one type of spectral data, the training data was specific to the application, and classified groups separately as a multiple binary classification problem ${ }^{48,49}$. Binary classifiers are not optimal for a large number of classes and are sensitive to class imbalances during training resulting in problems to identify all functional groups in a molecule or mixtures ${ }^{41,50}$. In this work, we present the first ML method, to our knowledge, that integrates FTIR and MS data to obtain a combined set of features as a multi-class, multi-label classification methodology. Our method predicts multiple functional groups for a given molecule in a database-free manner, as compared to identifying a molecule through peak matching or only identifying the major functional group in the molecule (Figure 1).

\section{Methods}

\section{Collection of training data}

We obtained both FTIR and MS spectra from standard reference spectra published by the United States National Institute for Science and Technology ${ }^{51}$ for 7,393 compounds and standardized these spectra using the procedure described in the supporting information under Standardization of IR spectra and Standardization of MS spectra. Please see the supporting information section titled Training and testing of neural networks for details on how the neural networks presented in this work are trained and optimized. 


\section{Assignment of functional groups}

We obtained IUPAC InChl strings for all compounds of interest by resolving the CAS number associated with the molecule using the PubChem API ${ }^{52}$. Then, RDKit ${ }^{53}$ performed substructure matching on each string via SMARTS strings to identify the presence of a predefined molecular topology. If a match for a functional group's SMARTS was found, then the compound was deemed a member of the given functional group, and each SMARTS string was tested independently. Therefore, multiple functional groups could be assigned to a single molecule. Initially, we picked functional groups common between those discussed in the previous works ${ }^{45,48,49}$.

After training our initial model and analysing the results (see Compounds that fail to be appropriately predicted show chemical patterns), we decided to add more functional groups to our model to attempt to improve our results. The SMARTS strings used for both models discussed in this work are shown in Table 1 and the distribution of functional groups are given in Figure S1.

\section{Calculation of a Molecular F1 metric}

Since the correct assignment of all functional groups in a single molecule is paramount to the analysis of organic reactions, we have devised a single metric to quantify the predictive capability of our models versus the performance on individual functional groups. Therefore, the focus of our optimization methodology is to create a model that maximizes this overall accuracy measure as opposed to the accuracies of individual functional groups. Similar to the concept of an F1 measure, this metric normalizes the performance when the classes (functional groups) are unbalanced. Hence, we have termed this metric as the 'Molecular F1 score' as it describes the success of the model on the whole molecule. This number is calculated for each molecule in the validation set by calculating a 'Molecular Precision' and 'Molecular Recall' value for the functional groups predicted for a given molecule. Precision is the number of functional groups predicted correctly (true positives) divided by the total number of functional groups predicted to be present (the sum true positives and false positives). Molecular recall is the number of functional groups predicted correctly divided by the total number of actual functional groups present in the molecule (the sum of true positives and false negatives). Similar to the calculation of an F1 score for given functional groups, the Molecular F1 is the harmonic mean of the Molecular Precision and Molecular Recall. The overall Molecular F1 score for a given validation set is the arithmetic 
mean of all Molecular F1 scores. The difference between the Molecular F1 and Functional Group F1 is illustrated in Figure 2.

\section{Calculation of a Molecular Perfection Rate metric}

While the knowledge of overall Molecular F1 score is useful for comparing models to one another, it does not represent the more stringent criterion of whether a given method produces all functional groups within a given molecule without error. Therefore, we have devised a second metric termed 'Molecular Perfection Rate' to rigorously measure the accuracy of our model on a per molecule basis. To calculate this metric, we compare the known functional groups to the predicted functional groups. If the predicted functional groups perfectly match the defined functional groups of the target molecule, then the molecule prediction pair is assigned a Molecular Perfection of 1; otherwise, it is assigned a Molecular Perfection of 0 . The 'Molecular Perfection Rate' for each validation set is calculated as the sum of all individual 'Perfections' values divided by the total number of molecules. This metric can also represent the percentage of all molecules with a Molecular F1 score of 1.0, as shown in Figure 2.

\section{Results and Discussion}

Multi-layer perceptron neural networks outperform Random Forest classifiers. We performed an initial computational experiment to determine the choice of machine learning method with the best performance to identify functional groups without doing extensive model optimization. We selected Random Forest (RF) and Multi-layered Perceptron (MLP) to test on IR spectra to determine if there was a need for using neural networks (MLP) as compared to ensemble methods (RF). An unoptimized MLP consistently outperformed RF models (Figure S2) with an average functional group F1-score of 0.771 for the MLP model compared to 0.650 for RF (see Table S1, S2 for F1-score of each model and Figure $\mathbf{2}$ and the experimental section for definitions of F1 score). We trained the MLP to predict all functional groups simultaneously as one multilabel classifier. In order to evaluate the effect of transfer learning that has been previously done for MLP ${ }^{40,41,50}$, we also evaluated 13 binary classifiers in addition to the 1 multi-label network. The binary classifier approach did not improve the performance of the MLP model significantly as these models only produced an improvement in functional group F1 score of 0.006 over the 
multi-label model, suggesting that transfer learning is not a significant factor in the multi-label network.

MS data addition improves the prediction of specific functional groups. Our MLP model trained on IR data performs well on alkanes, ketones, arenes, carboxylic acids, and esters (average validation F1-score of 0.926 ) but it did not perform at par to predict nitriles, amines, amides, and acyl halides with an average validation F1-score of 0.663 (Figure S3a, Table S3). We included the chemical features captured by mass spectrometry (MS) to augment the MLP-IR model (Figure S3a) to address these problematic functional groups. First, we trained an MLP model only on MS data to investigate its predictive capacity for functional groups (Figure S3b, Table S4). The difference between the F1 scores of the training set compared to the validation set indicates that MS data needs other models to generalize for consistent performance compared to IR data using MLP (Tables S3, S4). Similar to the MLP-IR model, the MLP-MS model performed well with more data for a given functional group (e.g. alkanes, arenes, alkyl halides), and poorly when fewer data were available (e.g. acyl halides, amides, and amines). Next, we investigated if combining IR and MS data could improve de novo prediction of functional by concatenating spectral data features into an IR+MS model (see experimental section). Table S5 shows the training and validation F1scores and Table $\mathbf{S} 6$ show the 5-fold result of the MLP model. The improvement of the IR+MS model over the IR model is presented as Figure S3c, and the direct F1 scores are shown in Figure S3d with an average improvement of 0.024 overall functional groups. However, combining IR and MS data results in a substantial increase in validation F1 scores for the nitrile, alkene, and alkyl halide functional groups with improvements of $0.124,0.048$, and 0.061 respectively. The amide functional group remains unchanged as the F1 score of 0.563 is the as the MLP-IR model. The improvement of alkyl halides (Figure S3c) may appear to match chemical intuition given the distinct pattern of halogen isotopes observed with MS. However, this conclusion is not supported by the architecture of an MLP model as each input neuron is independent. Future work incorporating the differences in abundance peaks instead of raw values may improve the performance of the MS only model. 


\section{Multiple functional groups prediction in a single compound present a second optimization}

problem. Analysis of the receiver operator characteristic (ROC) plots (Figure S4) shows that at $1 \%$ of the false-positive rate, the model identifies over $80 \%$ of the true positive functional groups. Therefore, we used a dynamic threshold for each functional group to determine the presence of a functional group in the molecule. This threshold is calculated to maximize the functional group F1 score for the training set after training is complete. While the ability of the model to predict the presence of a particular functional group is important for evaluating the performance of the model, a metric better suited for the study of chemistry and essential for autonomous instrumentation will be to measure the performance to prediction all functional groups in a given molecule. Therefore, we have introduced new metrics, such as the 'Molecular F1 score (MF1)' and the 'Molecular Perfection Rate (MPR)' (see Figure 2 and the methods section for more details) and optimized our models for the IR and IR+MS data. After optimization, the IR+MS model was able to perform on par or better than the optimized combined IR for the majority of functional groups (Figure 3). The resulting models have comparable average MPRs (72.5\% vs 74.9\%) and MF1s (0.923 vs 0.931) for IR and IR+MS respectively (see Tables S7, S8). The hyperparameters for these models are given in the supporting information under Details of the neural networks.

Guided backpropagation of the MLP model shows known IR and chemical patterns. We performed guided backpropagation on the optimized MLP-IR model for molecules that were both predicted with an MPR of 1 and has the greatest activation in the neuron corresponding to the respective functional group (Figure 4). Several backpropagation plots reveal a known chemical association between peaks in IR spectroscopy and functional group assignment. This is encouraging as the model was trained without any 'expert' or chemical information about the location of the peaks corresponding to each functional group. Specifically, we discuss several functional group cases for our selected set of molecules. The alkane functional group backpropagation shows the use of peaks near $3000 \mathrm{~cm}^{-1}$, matching the known location of alkane $\mathrm{CH}$ peaks tabulated in the literature. The remaining peaks, however, do not provide any additional chemical intuition with regards to the alkane functional group. Aromatic compounds 
are identified by a peak between $1400-1600 \mathrm{~cm}^{-1}$, and the model selected peaks within this region. In addition, the model was able to identify the alkene bending motion around $900 \mathrm{~cm}^{-1}$. A C-O stretch is typically observed around $1150 \mathrm{~cm}^{-1}$, and the backpropagation plots for carboxylic acids, alcohols, and esters indicate a peak in this region is used by our model for each of these functional groups. Additionally, a strong $\mathrm{C}=\mathrm{O}$ peak is typically observed for carbonyl compounds near $1600 \mathrm{~cm}^{-1}$, but the model only placed importance on this peak for the amide functional group. The example alcohol compound contained both an alcohol group and a carboxylic acid, and the model ignored the $\mathrm{C}=\mathrm{O}$ in the prediction of the alcohol, instead placing importance on peaks corresponding to the $\mathrm{O}-\mathrm{H}$ stretch near $3500 \mathrm{~cm}^{-1}$. These results show that the model reproduces the 'known chemistry' of functional group features without explicit input of peak to functional group relationships.

However, from our chosen set of molecules with MPR of 1, none of the backpropagation plots revealed any chemically significant characteristics for alkynes, amines, ketones, alkyl halides, and acyl halides. Instead, it appears that these functional groups are identified by the lack of sharp peaks in various regions of the spectra. This observation is interesting as the functional group F1 for these groups are relatively high. While nitrile groups have the lowest performance, the model was able to identify the $2210-2260 \mathrm{~cm}^{-1}$ band that is characteristic of this functional group. For the amine functional group, the model places high importance on a peak around $1550-1640 \mathrm{~cm}^{-}$ 1. Although this may appear to indicate learned chemistry since the known N-H bending in this region, it also conflicts with the $\mathrm{N}-\mathrm{O}$ bend of a nitro group. This observation may explain the reason our model misclassifies many nitro compounds as amides. Fortunately, there is a second $\mathrm{N}-\mathrm{O}$ bend present which may rectify this issue if we include nitro groups to the model separately.

Next, we investigated the compounds with at least one incorrect functional group prediction $(M P R=0)$ provided in Listing S1. There are noticeable patterns of functional group types present in the set of failures. One example is nitro groups, which appear over 20 times in the failed compounds. This group is of interest as it is characterized by two strong bands which overlap with bending modes in alkane and amides functional groups. Many of these nitro compounds 
are misclassified as amides or alkanes and this observation partially explains the poor performance of amide functional groups shown in Figure $\mathbf{3 d}$. Although it is discouraging to note that the model was unable to 'ignore' these peaks, the low count of amides present in the dataset may attribute to this poor performance.

Additional functional groups classification does not affect the model performance of the original definitions. In the previous section, we show that some functional groups explicitly trained in the MLP model were incorrectly classified due to overlapping peaks belonging to functional groups that were not included in our original set of functional group types. We hypothesized that the separate classification of the "overlapping" functional groups could affect the performance of our model. To test this hypothesis, we introduced the 'nitro,' 'ether,' and 'aldehyde' groups to the model. The 'nitro' group has significant overlap with the nitrile group (see the previous section), while the 'ether' group did not have peak values which overlapped with other functional groups in our previous definition. Another limitation of our model is the inability to distinguish methyl groups from other alkane functional groups. We propose that this is possible due to the lack of a $\mathrm{C}-\mathrm{C}$ stretch in methyl groups and methyl groups contain characteristic peaks not present in other alkane groups (i.e. the $\mathrm{CH} 3$ bend). In the NIST dataset many alkyl halides are present which do not contain any $\mathrm{C}-\mathrm{H}$ bonds as all hydrogens in the molecule have been halogenated. Due to the large size of the alkane functional group in the training set, we hypothesize that splitting the alkane group into methyl and 'other' alkanes will not result in a large decrease in performance. Therefore, we decided to subdivide the 'alkane' group into 'methyl,' and 'other' alkanes as these groups performed the best out of all other groups in the original model.

Figures 5a-c show the results of these two hypotheses with details presented in Figure S5 and Tables S9, S10. The relatively high F1 scores for the 'methyl' (0.932) and 'other' alkane (0.936) groups support our hypothesis that sub-division of the original alkane definition does not decrease performance. Figures 5a-b also suggest that our hypothesis to improve low performance of functional groups by the introduction of new functional groups for both IR and 
IR+MS MLP model is incorrect (compare Tables S9, S10 with Tables S3, S5). Although the nitrile and amide groups do not show improvement after the introduction of the nitro and ether groups as the F1 score for nitriles decreased by 0.019 and increased by 0.032 , the new groups perform well as compared to the original problematic groups ( 0.932 for nitro groups and 0.923 for ethers). This suggests that the addition of new functional groups does not cause a significant loss in F1 score for other groups. Therefore, we speculate that more complex groups could be added to the model to provide detailed structural information, such as a model to identify heterocyclic aromatic rings from rings comprised of only carbon. While further subdivision of functional groups is beyond the scope of this work, they present a potential extension of this work towards realization of autonomous instrumentation that results in minimal manual intervention.

Number of functional group predictions affects molecular perfection rate. We hypothesized that our stringent metric of MPR was affected by the increase in the number of functional group predictions for a given model. To test this hypothesis, we have created synthetic models based on the accuracies of each functional group from the trained IR+MS model (see Synthetic Models in the supporting information for more details). The machine learning model outperforms these synthetic models (Figures $\mathbf{5} \mathbf{d}$ and $\mathbf{S 5 d}$ ), indicating that increasing the number of functional groups does not decrease this metric more than what would be expected from the inclusion of additional functional groups alone. The overall conclusion of this section is encouraging as it suggests that more functional groups can be added to our model without hurting the model's ability to predict other functional groups. Values for the MPR and MF1 scores for the new functional group definitions are given in Tables S11 and S12.

We were also interested in the performance of our model on molecules with a differing number of functional groups. To do so, we calculated the molecular perfection rate for compounds with one through six functional groups, for the original set of functional groups and the new set of functional groups (results shown in Figure S6). Unfortunately, no definite conclusions can be made from this data as the original versus new functional group definitions follow very different patterns. However, the original set of functional groups outperforms the new set of definitions. 
This observation is likely due to the reduced accuracy of the new alkane due to the split into methyl and non-methyl groups as both have accuracies of $91 \%$ where the previous model had an accuracy of $95 \%$ (Figure $5 c$ ).

Encoding spectra data in latent space retains functional group prediction performance. Given the success of our MLP model in predicting functional groups using complete standardized spectra, we wished to investigate the ability of an autoencoder to reduce the spectra into a latent space. We trained a simple linear model for encoding the IR and MS spectra into a 256-length vector and decoding this vector back to the original spectra used to create the vector (see Figure 1). The 256-length vector was used to train a second network for multi-task functional group prediction. For individual functional groups, the autoencoder model performs similar to that of the original MLP model (F1 scores are given in Tables S13 and S14) The molecular performance of the autoencoder model is similar to that of the original MLP model (Figure 6) as the MPR for the autoencoder model is $62.6 \%$ and the MF1 score is 0.905 as compared to $65.2 \%$ and 0.912 for the original model (Tables S15 and S16). This reveals that the original spectra contain redundant features that relate IR and mass spectra. We plan to explore the use of this latent space for inverse design of molecules with combined spectral properties in future works.

Deep learning model trained on single compounds predicts functional groups in mixtures. The ability to identify all the functional groups in a mixture of compounds expands the applicability of our methodology. To our knowledge, we are the first group to report the ability of machine learning methods to classify mixtures of compounds using a model trained on single compounds. To validate our method on compound mixtures, we obtained the IR spectra of three different mixtures of molecules (raw spectra given in Figures S8-10) and predicted all the functional groups of the compounds in the mixture using our MLP-IR machine learning model (see Table 2). We stress the point that these spectra are obtained in our lab, are not part of the NIST dataset, and are obtained from instruments different than those used by the NIST as it is essential to validate a machine learning method for practical use in different laboratories. Since these spectra are external to the NIST webbook data, they constitute a 'test set' for our model. The compound 
mixtures were prepared by mixing two solid compounds and each mixture contained different set of functional groups. Performance metrics, such as molecular F1 score etc., described previously for single molecules are applied to a mixture of molecules by considering the set of all functional groups (a union of all functional groups present in the mixture). For mixture 1, our IRonly method correctly predicted 2 out of the 4 functional groups present in the mixture, and predicted an additional functional group not present in the mixture, yielding an MF1 score of 0.65 (Table 2). Given the resolution of spectral data, the lack of an O-H peak above $3500 \mathrm{~cm}^{-1}$ could also lead a human chemist to conclude that no carboxylic acid is present in the mixture (Figure S8). Additionally, the presence of a peak near $2940 \mathrm{~cm}^{-1}$ may lead a human to conclude that a methyl group is present in the mixture (Figure S8). For mixture 2, we obtain an MF1 score for the mixture of 0.80 as we correctly predict 2 out of the three functional groups present in the mixture and do not predict any additional functional groups. The only missed functional group is the amide group, which is known to be problematic in our model (functional group F1 score < 0.60 ) and the lack of a strong peak near $1650 \mathrm{~cm}^{-1}$ may contribute to a human's inability to identify this functional group (Figure S9). For mixture 3, our method correctly predicts 3 out of the 6 functional groups in the mixture and does not predict any additional groups in the mixture, yielding a molecular $F 1$ score of 0.67 . The model was not able to identify a methyl group and a human may make the same mistake given the lack a peak near $2940 \mathrm{~cm}^{-1}$ (Figure S10). The model also failed to predict the presence of a nitro group and the presence of an ether, potentially due to the peaks corresponding to these groups overlapping with other peaks in the aromatic region of the spectra. Our results show that the deep learning model trained on single compound spectra can give reasonable performance to predict functional groups for mixtures of compounds. Future work entails training on compound mixture spectral data along with using other deep learning architectures, such as Generative Adversarial Networks. This is essential for correctly estimating the limitations of machine learning models for adoption in industry for autonomous instrumentation.

\section{Conclusion}


We present a machine learning method for de novo prediction of functional groups using a combination of IR and MS data. We introduce two new metrics apart from functional group F1score, namely, molecular F1-score and molecular perfection rate for practical use of our models. Our results show that, in general, the IR data is more consistent for predicting functional groups than MS data, a conclusion backed by chemical intuition. However, several functional group predictions benefit from the inclusion of MS data. Additionally, our model architecture is more optimal for analysis of IR data due to the continuous nature of these spectra, and the mathematical structure of an MLP model. Our model's performance is not affected by the number of functional groups present in the training data and it predicted all the functional groups consistently across all metrics. Moreover, several known chemical patterns in the spectra were identified as features for the model to identify common functional groups without any expert training of the system. We conclude that a multi-class, multi-label perspective is apt for further studies which may combine differing spectroscopic data types that may reveal unknown features useful for the identification of compounds. We show that our approach for functional group predictions is flexible as it can be extended to introduce new or sub-divide existing functional groups without affecting performance of original functional group definitions. Furthermore, reducing chemical spectral data in a latent space does affect model performance to predict functional groups but can be used for inverse design of molecules based on combination of spectral properties. Finally, we have verified that our model also produces reasonable results for a mixture of compounds containing multiple, different, functional groups.

\section{Data Availability}

All code required to generate the models presented in this work is available upon request. The NIST Webbook data is copyrighted by NIST and must be obtained from this organization accordingly. 


\section{References}

1 H. C. Kolb and K. B. Sharpless, Drug Discov. Today, 2003, 8, 1128-1137.

2 S. Chatani, D. P. Nair and C. N. Bowman, Polym. Chem., 2013, 4, 1048-1055.

3 V. L. S. Freitas and M. D. M. C. Ribeiro da Silva, Molecules, 2018, 23, 2962.

4 B. D. Marshall and C. P. Bokis, Fluid Phase Equilib., 2018, 478, 34-41.

5 Y. M. Dai, Z. P. Zhu, C. Zhong, Y. F. Zhang, J. L. Zeng and L. Xun, J. Mol. Graph. Model., 2013, 44, 113-119.

6 M. Withnall, H. Chen and I. V. Tetko, ChemMedChem, 2018, 13, 599-606.

7 T. Takei, M. Nakada, N. Yoshikawa, Y. Hiroe and H. Yoshida, J. Therm. Anal. Calorim., 2016, 123, 1787-1794.

8 P. Y. Bruice, Essential Organic Chemistry, Pearson, Upper Saddle Reiver, New Jersey, 3rd edn., 2016.

9 F. B. Cordeiro, C. R. Ferreira, T. J. P. Sobreira, K. E. Yannell, A. K. Jarmusch, A. P. Cedenho, E. G. Lo Turco and R. G. Cooks, Rapid Commun. Mass Spectrom., 2017, 31, 1462-1470.

10 A. Minai-Tehrani, N. Jafarzadeh and K. Gilany, Andrologia, 2016, 48, 609-616.

11 A. V. Ewing and S. G. Kazarian, Analyst, 2017, 142, 257-272.

12 R. Risoluti, S. Materazzi, A. Gregori and L. Ripani, Talanta, 2016, 153, 407-413.

13 J. Manheim, K. C. Doty, G. McLaughlin and I. K. Lednev, Appl. Spectrosc., 2016, 70, 11091117.

14 P. T. Anastas, M. Fontalvo Gómez, B. Johnson Restrepo, T. Stelzer and R. J. Romañach, in Handbook of Green Chemistry, Wiley-VCH Verlag GmbH \& Co. KGaA, Weinheim, Germany, 2019, pp. 257-288.

15 M. J. Baker, J. Trevisan, P. Bassan, R. Bhargava, H. J. Butler, K. M. Dorling, P. R. Fielden, S. W. Fogarty, N. J. Fullwood, K. A. Heys, C. Hughes, P. Lasch, P. L. Martin-hirsch, B. Obinaju, G. D. Sockalingum, J. Sulé-suso and R. J. Strong, Nat. Protoc., 2014, 9, 1771-1791.

16 J. Li, D. B. Hibbert, S. Fuller and G. Vaughn, Chemom. Intell. Lab. Syst., 2006, 82, 50-58.

17 J. Griffiths, Anal. Chem., 2008, 80, 5678-5683.

18 K. Dührkop, H. Shen, M. Meusel, J. Rousu and S. Böcker, Proc. Natl. Acad. Sci., 2015, 112, $12580-12585$. 
19 T. Kind, H. Tsugawa, T. Cajka, Y. Ma, Z. Lai, S. S. Mehta, G. Wohlgemuth, D. K. Barupal, M. R. Showalter, M. Arita and O. Fiehn, Mass Spectrom. Rev., 2018, 37, 513-532.

20 E. L. Schymanski, J. Jeon, R. Gulde, K. Fenner, M. Ruff, H. P. Singer and J. Hollender, Environ. Sci. Technol., 2014, 48, 2097-2098.

21 F. Hufsky and S. Böcker, Mass Spectrom. Rev., 2017, 36, 624-633.

22 J. Li, S. G. Ballmer, E. P. Gillis, S. Fujii, M. J. Schmidt, A. M. E. Palazzolo, J. W. Lehmann, G. F. Morehouse and M. D. Burke, Org. Synth., 2015, 347, 1221.

23 J. M. Granda, L. Donina, V. Dragone, D. L. Long and L. Cronin, Nature, 2018, 559, 377381.

24 D. T. Ahneman, J. G. Estrada, S. Lin, S. D. Dreher and A. G. Doyle, Science (80-. )., 2018, 360, 186-190.

25 C. Wang and Y. Zhang, J. Comput. Chem., 2017, 38, 169-177.

26 F. Pereira, K. Xiao, D. A. R. S. Latino, C. Wu, Q. Zhang and J. Aires-De-Sousa, J. Chem. Inf. Model., 2017, 57, 11-21.

27 J. Ma, R. P. Sheridan, A. Liaw, G. E. Dahl and V. Svetnik, J. Chem. Inf. Model., 2015, 55, 263-274.

28 F. A. Faber, L. Hutchison, B. Huang, J. Gilmer, S. S. Schoenholz, G. E. Dahl, O. Vinyals, S. Kearnes, P. F. Riley and O. A. Von Lilienfeld, J. Chem. Theory Comput., 2017, 13, 52555264.

29 J. N. Wei, D. Duvenaud, A. Alán Aspuru-Guzik and A. Aspuru-Guzik, ACS Cent. Sci., 2016, 2, 725-732.

30 B. Sanchez-Lengeling, C. Outeiral, G. L. Guimaraes and A. Aspuru-Guzik, ChemRxiv, 2017, 1-18.

31 M. Benhenda, , DOI:1708.08227v3.

32 E. Putin, A. Asadulaev, Y. Ivanenkov, V. Aladinskiy, B. Sanchez-Lengeling, A. Aspuru-Guzik and A. Zhavoronkov, J. Chem. Inf. Model., 2018, 58, 1194-1204.

33 G. L. Guimaraes, B. Sanchez-Lengeling, C. Outeiral, P. L. C. Farias and A. Aspuru-Guzik, ChemRxiv, 2017, 1-7.

34 A. Kadurin, S. Nikolenko, K. Khrabrov, A. Aliper and A. Zhavoronkov, Mol. Pharm., 2017, 
14, 3098-3104.

35 B. Liu, B. Ramsundar, P. Kawthekar, J. Shi, J. Gomes, Q. Luu Nguyen, S. Ho, J. Sloane, P. Wender and V. Pande, ACS Cent. Sci., 2017, 3, 1103-1113.

36 M. H. S. Segler, T. Kogej, C. Tyrchan and M. P. Waller, ACS Cent. Sci., 2018, 4, 120-131.

37 Z. Xu, S. Wang, F. Zhu and J. Huang, in Proceedings of the 8th ACM International Conference on Bioinformatics, Computational Biology, and Health Informatics - ACM$B C B$ '17, ACM Press, New York, New York, USA, 2017, pp. 285-294.

38 Y. Jing, Y. Bian, Z. Hu, L. Wang and X.-Q. S. Xie, AAPS J., 2018, 20, 58.

39 R. Gómez-Bombarelli, J. N. Wei, D. Duvenaud, J. M. Hernández-Lobato, B. SánchezLengeling, D. Sheberla, J. Aguilera-Iparraguirre, T. D. Hirzel, R. P. Adams and A. AspuruGuzik, ACS Cent. Sci., 2018, 4, 268-276.

40 T. B. Hughes, G. P. Miller and S. J. Swamidass, Chem. Res. Toxicol., 2015, 28, 797-809.

41 T. B. Hughes, N. Le Dang, G. P. Miller and S. J. Swamidass, ACS Cent. Sci., 2016, 2, 529537.

42 H. Altae-Tran, B. Ramsundar, A. S. Pappu and V. Pande, ACS Cent. Sci., 2017, 3, 283-293.

43 S. Kearnes, K. McCloskey, M. Berndl, V. Pande and P. Riley, J. Comput. Aided. Mol. Des., 2016, 30, 595-608.

44 M. Krenn, F. Häse, A. Nigam, P. Friederich and A. Aspuru-Guzik, .

45 R. Nalla, R. Pinge, M. Narwaria and B. Chaudhury, in Proceedings of the ACM India Joint International Conference on Data Science and Management of Data-CoDS-COMAD '18, 2018, pp. 201-209.

46 S. Barbon, A. P. A. da Costa Barbon, R. G. Mantovani and D. F. Barbin, J. Spectrosc., 2018, 2018, 1-12.

47 W. Fu and W. S. Hopkins, J. Phys. Chem. A, 2018, 122, 167-171.

48 R. J. Fessenden and L. Györgyi, J. Chem. Soc., Perkin Trans. 2, 1991, 1755-1762.

49 E. W. Robb and M. E. Munk, Mikrochim. Acta, 1990, 100, 131-155.

50 S. J. Pan and Q. Yang, IEEE Trans. Knowl. Data Eng., 2010, 22, 1345-1359.

51 P. J. Linstrom and W. G. Mallard, Eds., NIST Chemistry WebBook, NIST Standard Reference Database Number 69, National Institute of Standards and Technology, Gaithersburg MD, 
20899, 2005.

52 S. Kim, P. A. Thiessen, E. E. Bolton, J. Chen, G. Fu, A. Gindulyte, L. Han, J. He, S. He, B. A. Shoemaker, J. Wang, B. Yu, J. Zhang and S. H. Bryant, Nucleic Acids Res., , DOI:10.1093/nar/gkv951.

53 G. A. Landrum, 2011. 
Figures

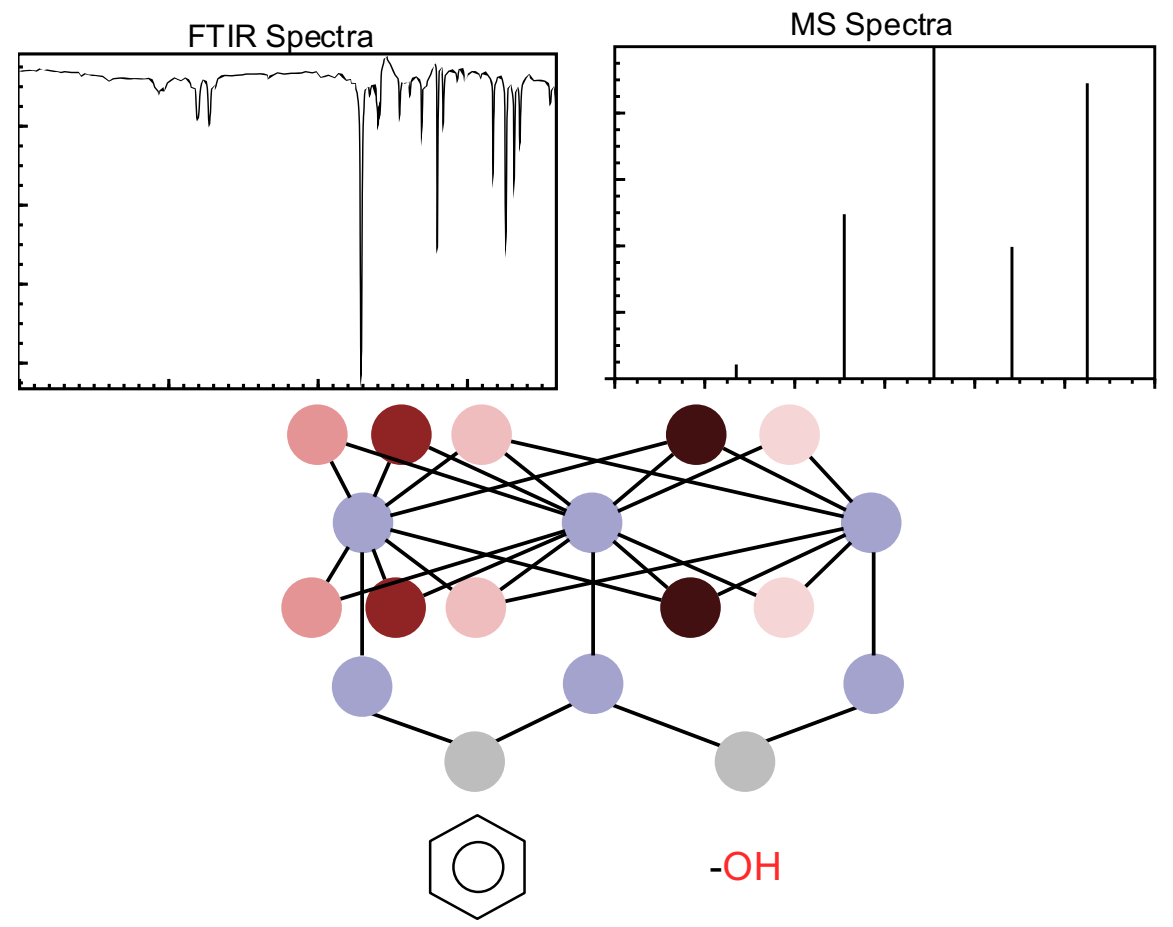

TOC Figure

A new multi-label deep neural network architecture is used to combine Infrared (IR) and mass spectra, trained on single compounds to predict functional groups, and experimentally validated on complex mixtures. 


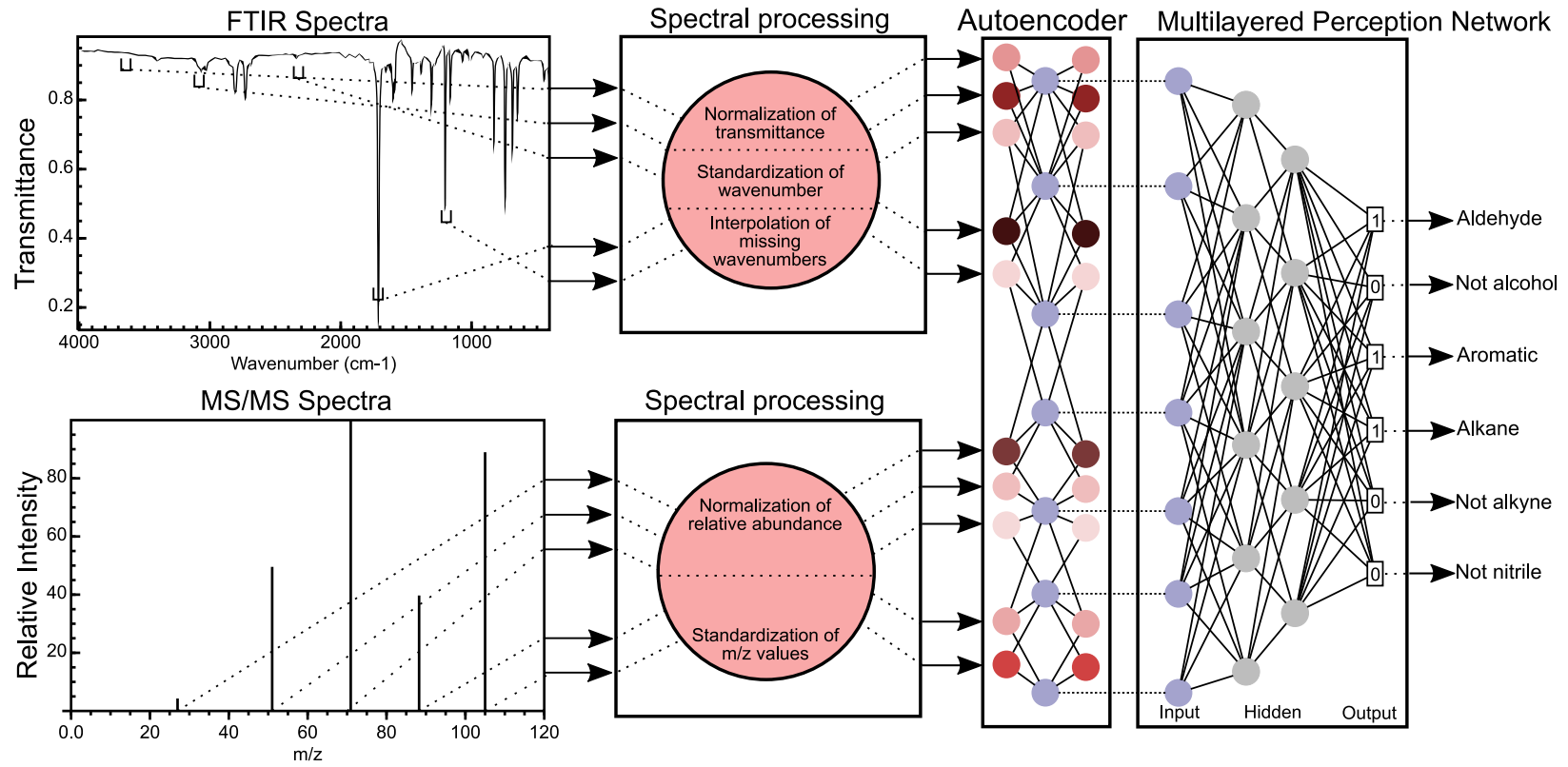

Figure 1. Overview of the MLP methodology for the classification of functional groups using FTIR and MS data. FTIR spectra are processed as to normalize the transmittance of the spectra and discretize the wavenumber numbers (creating wavenumber bins), thereby standardizing the wavenumbers for all FTIR spectra. Missing wavenumber bins in each spectrum are interpolated using B-Splines. A similar process is used for mass spectra data with the exception that no interpolation is performed. The normalized transmittance in all bins is encoded into a latent space by an autoencoder network and This latent space this then used to predict the functional group of a molecule. 

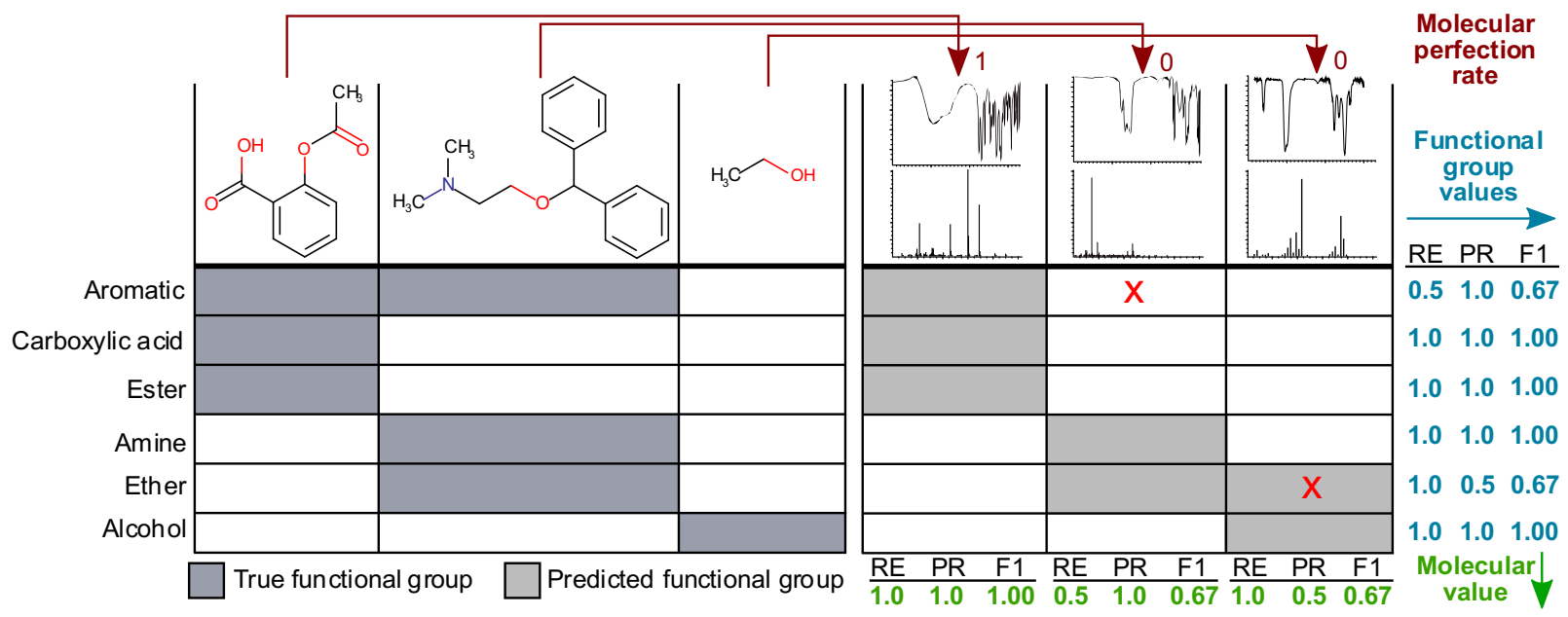

Figure 2: The left-hand side of the figure depicts the 'true' functional groups present in the example molecules, and the right-hand side are example predictions of the molecules functional groups given only their IR and MS spectra. Sample calculations for functional group F1, molecular F1, and molecular F1 score are given in the figure. Here, RE is short for 'recall' and PR is short for 'precision.' 
a
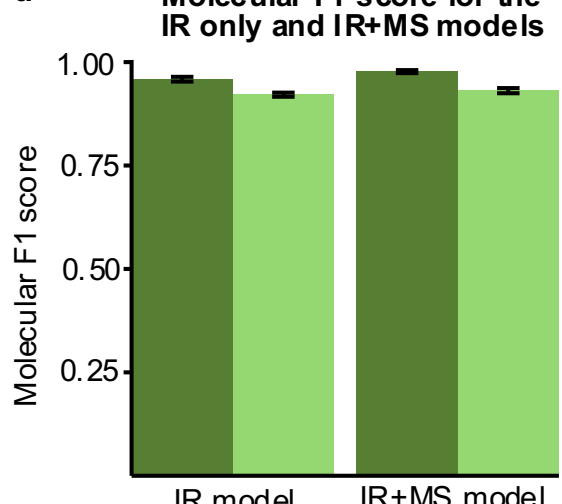

Validation F1

C Functional group F1 scores for the IR only model

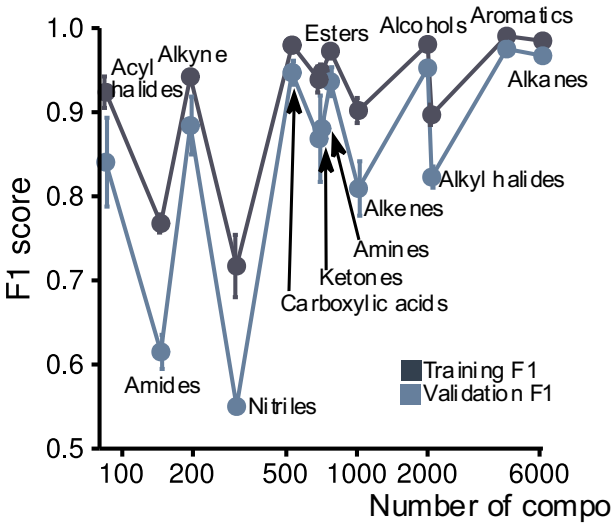

b Molecular perfection rate for the IR only and IR+MS models

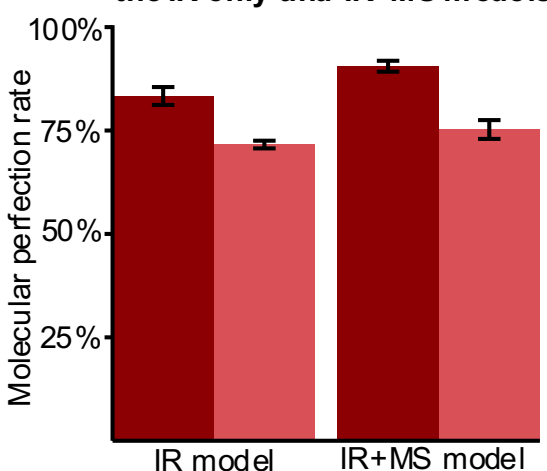

Training perfection Validation perfection

d Functional group F1 scores for the IR+MS model

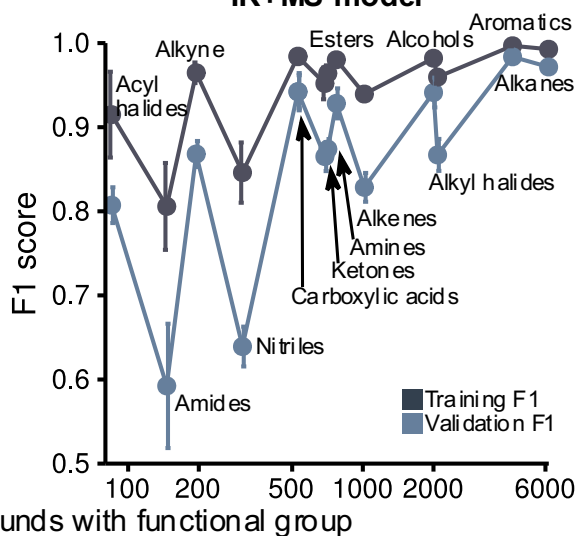

Figure 3: (a) The molecular F1 score for training and validation over the 5 folds is shown for both the optimized IR only and IR+MS models. The error bars indicate the standard deviation over the folds. (b) The molecular perfection for training and validation over 5 folds is shown for both the optimized IR only and IR+MS models. (c) The F1 score of the optimized IR only model plotted against the number of occurrences of that functional group. (d) The F1 score of the optimized IR+MS model plotted against the number of occurrences of that functional group. 

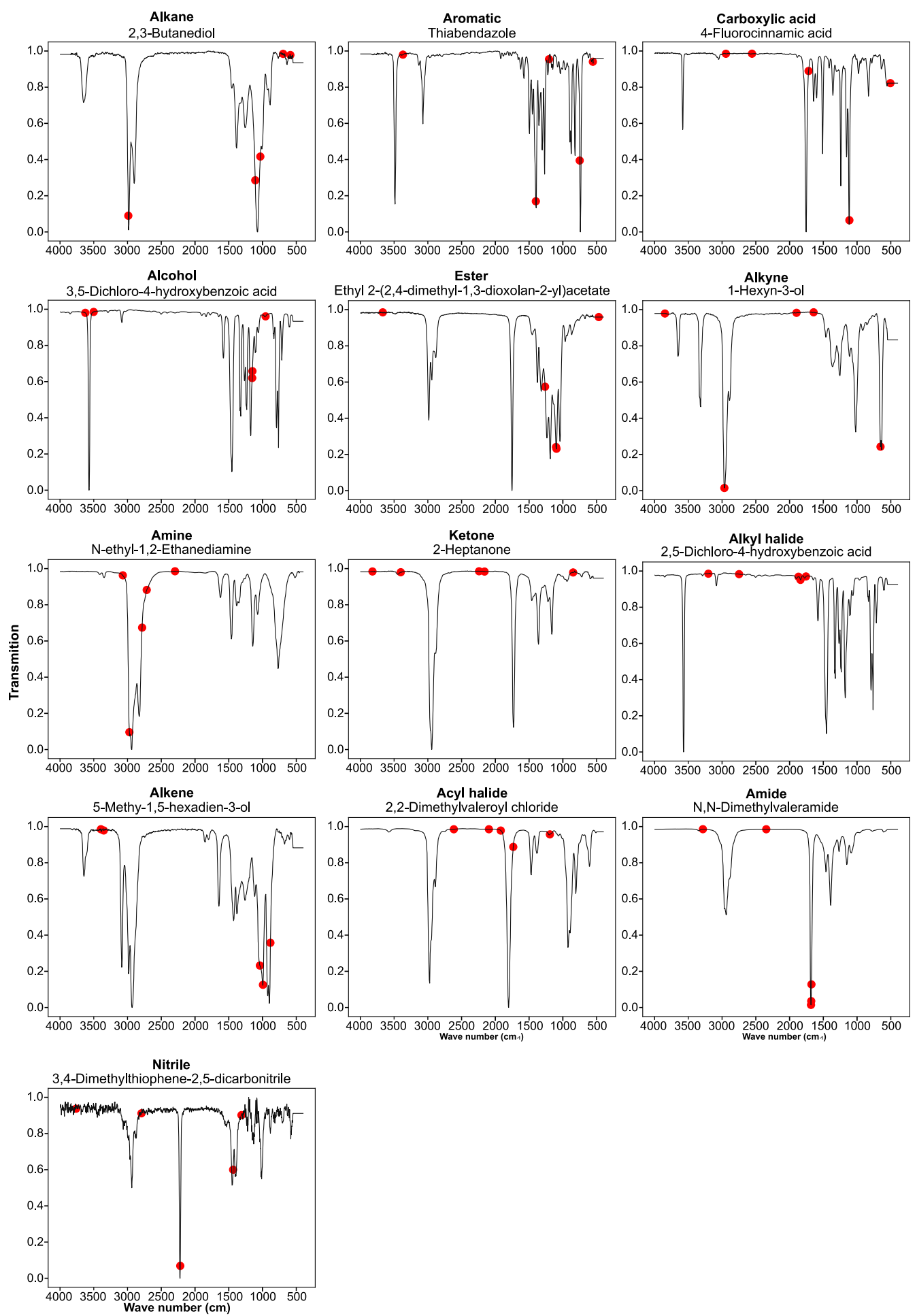

Figure 4: Backpropagation analysis for all 13 functional groups was performed to identify the regions of the spectra responsible for the result given. These plots are listed above in order of decreasing F1 score for the optimized IR+MS model. 


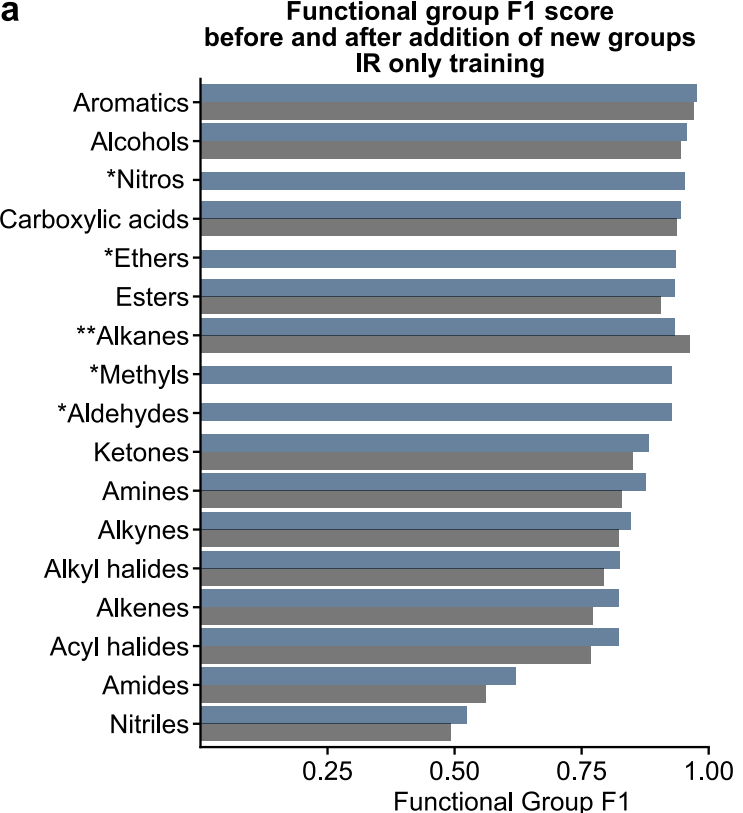

Performance with new groups Performance of the original model b

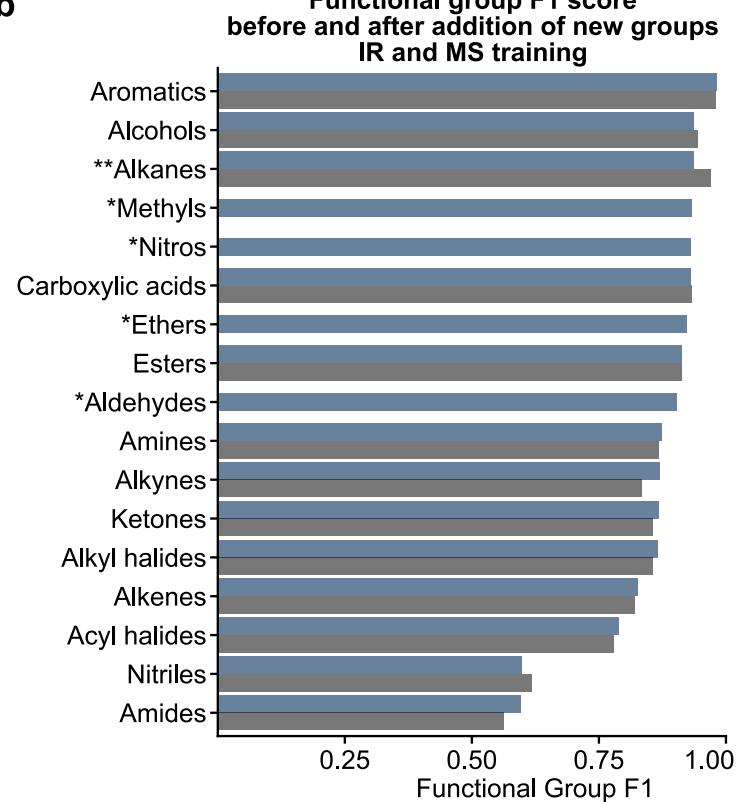

*New functional group ${ }^{* *}$ Redefined group
C

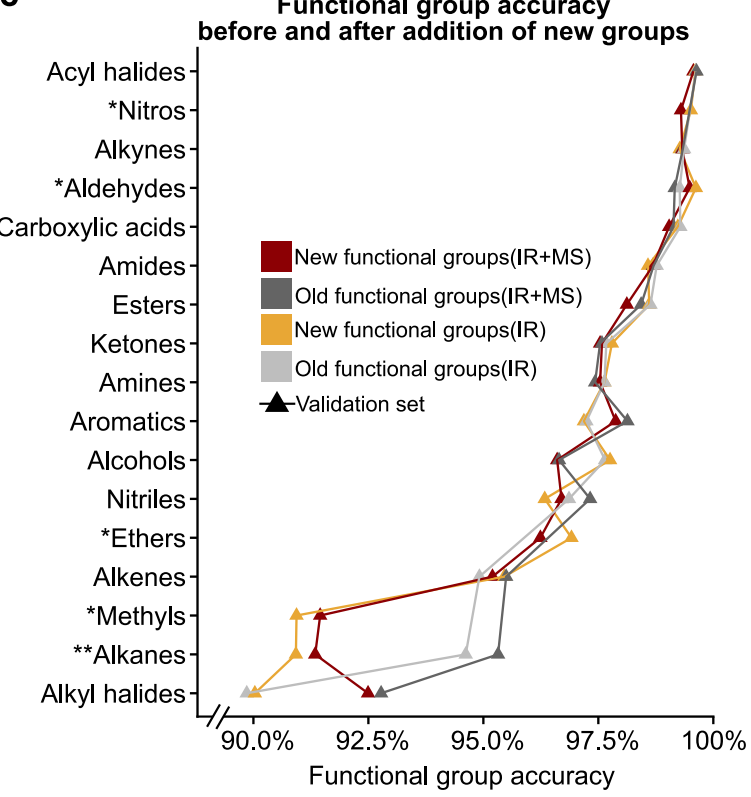

d

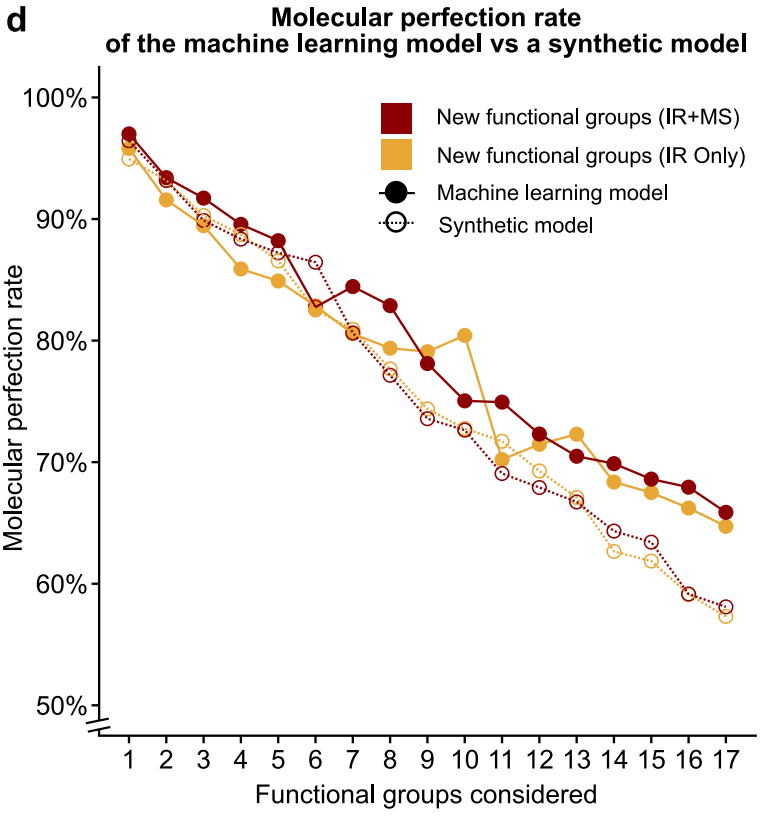

Figure 5: The bar plots given in (a) - (b) compare the functional group F1 scores for the original definitions of functional groups to the new definitions (see Table 1) showing that the addition of new additional functional groups does not have a significant impact on the previous functional groups. The line plot in (c) shows that the accuracy only decreases for the redefined functional group. The plot of molecular perfection rate in (d) compares the performance of the machine learning model to a synthetic model to show that the decrease in molecular perfection rate is expected as the number of functional groups increases. 
a

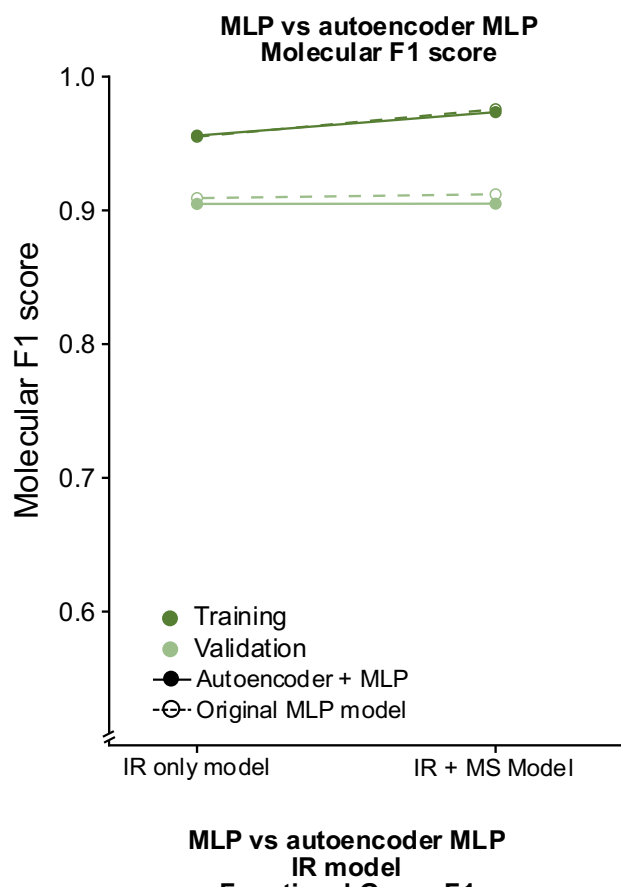

b

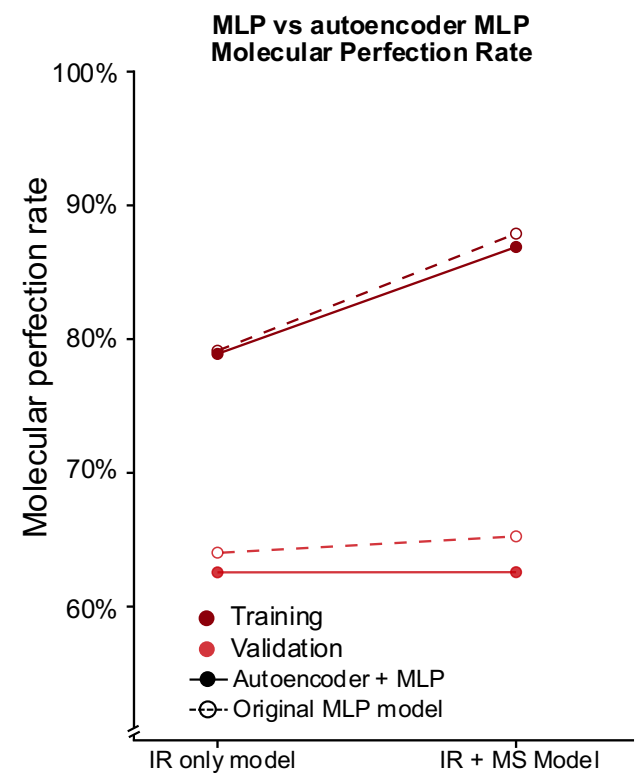

d

d

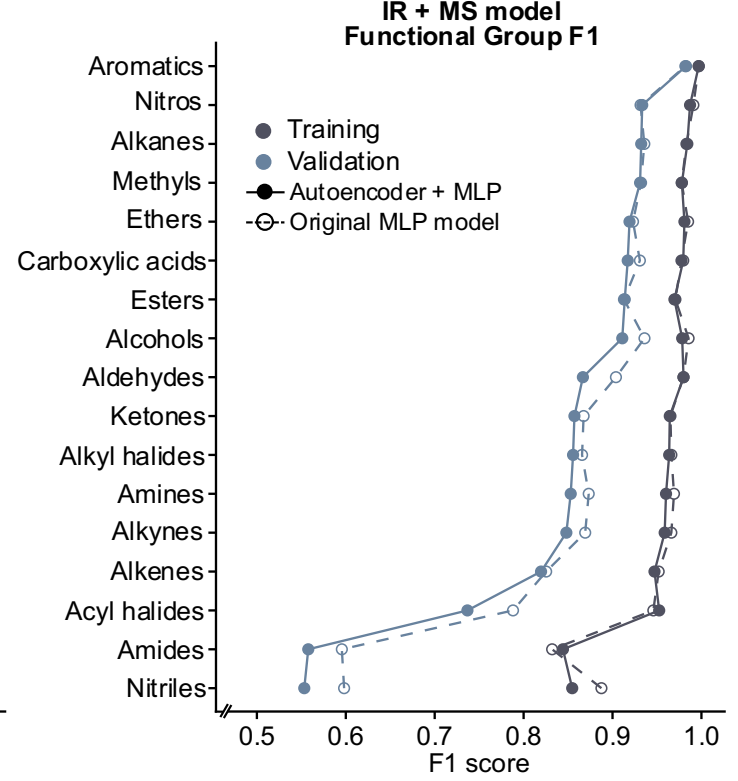

Figure 6: Comparison between the original MLP model and the autoencoder based model using the (a) molecular F1 metric and (b) molecular perfection rate are shown. Individual functional group F1 scores are provided for the IR only (c) and IR+MS (d) latent spaces. 


\section{Tables}

Table 1. SMARTS strings used to identify the presence of a functional group given the 2D topology of a molecule.

\begin{tabular}{ll}
\hline $\begin{array}{l}\text { Functional } \\
\text { group }\end{array}$ & Smarts String \\
\hline Alkane ${ }^{\mathrm{a}]}$ & {$[\mathrm{CX} 4]$} \\
Alkene & {$[\$([\mathrm{CX} 2]=[\mathrm{X} 2])]$} \\
Alkyne & {$[\$([\mathrm{CX} 2] \# \mathrm{C})]$} \\
Arene & {$[\mathrm{c}]$} \\
Ketone & {$[\# 6][\mathrm{CX} 3](=\mathrm{O})[\# 6]$} \\
Ester & {$[\# 6][\mathrm{CX} 3](=\mathrm{O})[\mathrm{OX} 2 \mathrm{HO}][\# 6]$} \\
Amide & {$[\mathrm{NX3}][\mathrm{CX} 3](=[\mathrm{OX} 1])[\# 6]$} \\
Carboxylic & {$[\mathrm{CX} 3](=\mathrm{O})[\mathrm{OX} 2 \mathrm{H} 1]$} \\
acid & \\
Alcohol & {$[\mathrm{CHX} 4][\mathrm{OX} 2 \mathrm{H}]$} \\
Amine & {$[\mathrm{NX} 3 ; \mathrm{H} 2, \mathrm{H} 1 ; ! \$(\mathrm{NC}=\mathrm{O})]$} \\
Nitrile & {$[\mathrm{NX} 1] \#[\mathrm{CX} 2]$} \\
Akyl halide & {$[\mathrm{CX} 4][\mathrm{F}, \mathrm{Cl}, \mathrm{Br}, \mathrm{I}]$} \\
Acyl halide & {$[\mathrm{CX} 3](=[\mathrm{OX} 1])[\mathrm{F}, \mathrm{Cl}, \mathrm{Br}, \mathrm{I}]$} \\
Ether $^{[\mathrm{b}]}$ & {$[\mathrm{OD} 2]([\# 6])[\# 6]$} \\
Nitro ${ }^{[\mathrm{b}]}$ & {$[\$([\mathrm{NX} 3](=\mathrm{O})=\mathrm{O}), \$([\mathrm{NX} 3+]($} \\
& $=\mathrm{O})[\mathrm{O}-])][! \# 8]$ \\
Methyl ${ }^{[\mathrm{b}]}$ & {$[\mathrm{CH} 3 X 4]$} \\
Alkane $^{[\mathrm{b}]}$ & {$[\mathrm{CX} 4 ; \mathrm{HO}, \mathrm{H} 1, \mathrm{H} 2]$} \\
\hline
\end{tabular}

[a] The alkane group is redefined in the second set of functional group definitions. [b] Groups only present in the second set of functional group definitions. 
Table 2. Mixtures of molecules used as a test set for the final model

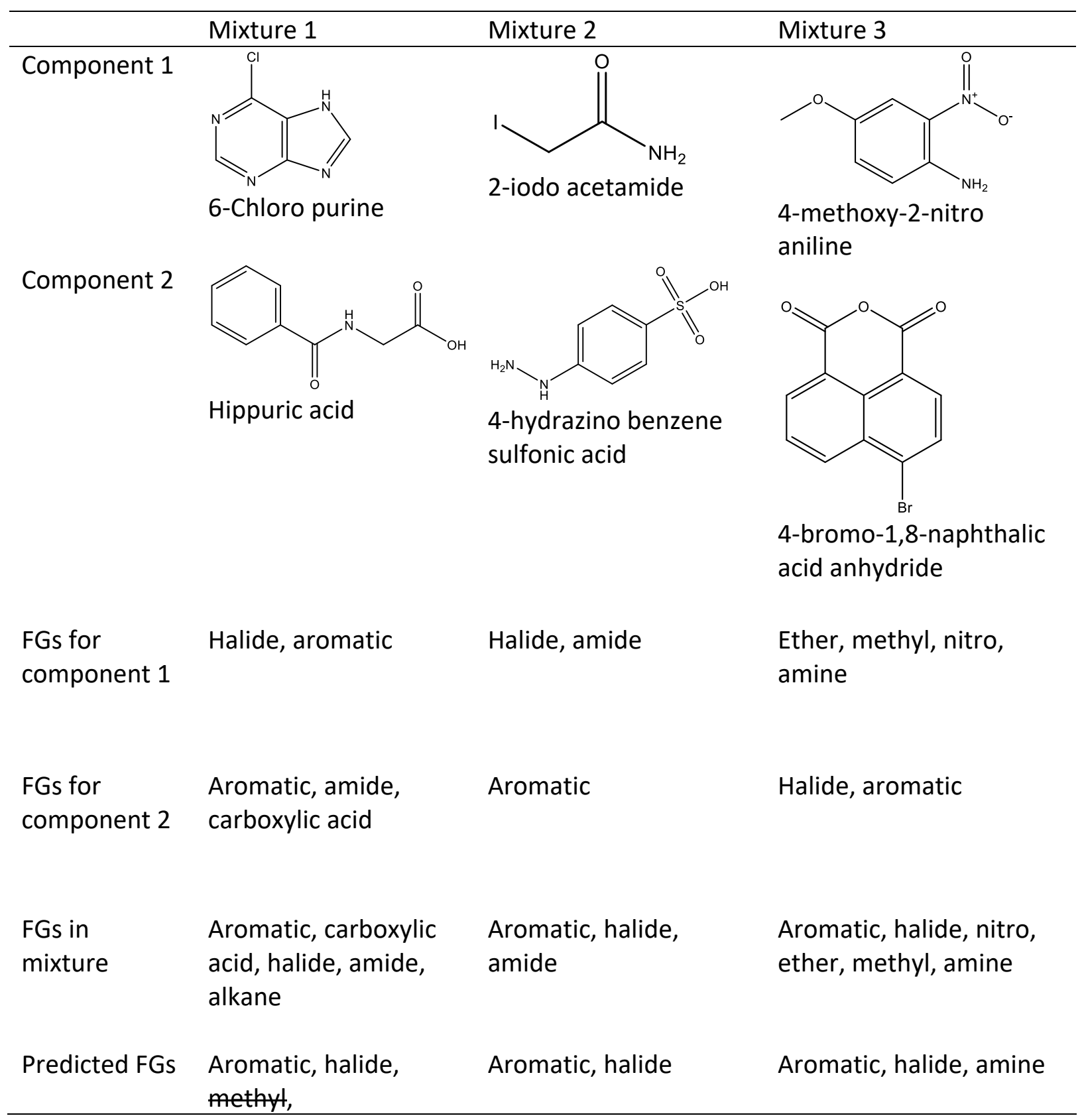


Other files 